\title{
Effect of pore connectivity on reflection amplitudes of an inhomogeneous wave in a composite porous solid saturated by two immiscible fluids
}

\author{
Neeru Bala ${ }^{1,3}$ and Ashish Arora ${ }^{2, *}$ \\ ${ }^{1}$ Department of Mathematics, DAV Institute of Engineering and Technology, Jalandhar 144 008, India. \\ ${ }^{2}$ Department of Mathematics, IKG Punjab Technical University, Kapurthala 144 603, India. \\ ${ }^{3}$ IKG Punjab Technical University, Kapurthala 144 603, Punjab, India. \\ *Corresponding author. e-mail: dr.ashish10@hotmail.com
}

MS received 16 January 2017; revised 13 September 2017; accepted 28 September 2017;

published online 29 May 2018

Present paper aims to study the phenomenon of reflection and transmission when an inhomogeneous wave strikes some discontinuity in a composite porous medium saturated by two immiscible viscous fluids. The incident wave splits into six reflected and six transmitted waves at the interface. All reflected and transmitted waves are inhomogeneous in nature with different directions of propagation vector and attenuation vector. A dimensionless parameter $\varsigma \in[0,1]$ is introduced to represent the extent of connection among the pores at the interface. Expression of Umov-Poynting vector is derived to obtain energy flux vector. Continuity of energy flux vector at the interface gives the required boundary conditions for the system. Connecting parameter $\varsigma$ is also employed in boundary conditions to model the partial connection of pores at the interstices of two media. For numerical discussion we consider a porous medium composed of sandstone and ice, saturated with oil and water. The effect of parameter $\varsigma$ and angle of incidence is determined numerically on the amplitude and the energy ratios of reflected and transmitted waves.

Keywords. Composite porous medium; reflection; transmission; inhomogeneous wave; pore connectivity; energy flux.

\section{Nomenclature}

$S_{\mathrm{f}} \quad$ Saturation of each fluid phase

$S_{s_{f}} \quad$ Fraction of each solid in composite matrix

$R_{11}, R_{22}$ Coefficients related to viscous drag

$A_{11}, A_{22}$ Coefficients related to inertial drag of first solid

$B_{11}, B_{22}$ Coefficients related to inertial drag of second solid

$A_{12} \quad$ Inertial coupling parameter connecting fluid phases

$G_{s_{\mathfrak{f}}} \quad$ Shear modulus of each solid phase

$k_{\beta} \quad$ Complex wave number of dilatational wave $k_{l} \quad$ Complex wave number of rotational wave

$w_{i}^{f}$, Normal component of drainage velocity of pore fluids

$\hat{a} \quad$ Unit normal vector to surface $\boldsymbol{S}$

$F, F^{\prime} \quad$ Time averaged energy flux along normal at interface in both half spaces

$T_{1}, T_{2} \quad$ Surface flow impedance for both fluids

$A_{m} \quad$ Attenuation vector of propagating waves

$P_{m} \quad$ Propagation vector of propagating waves

$A_{o} \quad$ Attenuation vector of incident wave

$P_{o} \quad$ Propagation vector of incident wave

$s \quad$ Slowness vector of a wave

$s_{x}, s_{z}$ Horizontal and vertical components of slowness 
$s_{m z} \quad$ Vertical slowness of propagating waves

$Z_{o} \quad$ Amplitude of incident wave

$Z_{m} \quad$ Amplitude of reflected waves

$Z_{m}^{\prime} \quad$ Amplitude of transmitted waves

$F_{m m} \quad$ Orthodox energy flux

$F_{m n} \quad$ Interference energy flux

$F_{o} \quad$ Energy flux due to incident wave

$E_{m n} \quad$ Energy ratios of reflected waves to incident wave

$E_{m n}^{\prime} \quad$ Energy ratios of transmitted waves to incident wave

$K_{\alpha} \quad$ Bulk modulus of $\alpha$ phase

$K_{s_{f}} \quad$ Drained bulk modulus of solids

$\mathbb{K}_{\mathfrak{f}} \quad$ Permeability of solid phases

$\mathfrak{T}_{s_{\mathfrak{f}}} \quad$ Tortuosity of solids

$K_{r_{f}} \quad$ Relative permeability of fluids

$f \quad$ Porosity of medium

$p_{c} \quad$ Capillary pressure difference between fluids

$\mathfrak{h}, \mathfrak{n}, \mathfrak{g} \quad$ Fitting parameters

\section{Greek Symbols}

$\rho_{\alpha} \quad$ Density for the phase $\alpha$

$\theta_{\alpha} \quad$ Volume fraction for the phase $\alpha$

$\sigma^{\alpha} \quad$ Partial stress for the phase $\alpha$

$\sigma_{i j}^{\alpha} \quad$ Stress tensor of each phase

$\epsilon_{i j}^{\alpha} \quad$ Strain tensor of each phase

$\Gamma^{\mathfrak{f}} \quad$ Coefficients related to effective stress

$\sigma \quad$ Sum of normal stresses of each phase

$\varsigma \quad$ A parameter representing connection pores at interface

$\theta_{o} \quad$ Angle of incidence

$\Omega \quad$ Upper half space

$\Omega^{\prime} \quad$ Lower half space

$\eta_{\mathfrak{f}} \quad$ Viscosity of fluid phases

$\gamma_{m} \quad$ Angle of attenuation of propagating waves

$\gamma_{o} \quad$ Angle of attenuation of incident wave

\section{Introduction}

The study of reflection and transmission of a wave at an interface in a porous medium is not only of academic interest but has considerable applications in many fields like soil mechanics, earthquake engineering and seismic exploration (Blum et al. 2004; Adamo et al. 2004; Gorodetskaya 2005). Rocks are porous in nature and generally composed of different materials. Wave propagating through these rocks get scattered when encountered with some discontinuity. The study of reflection and refraction amplitudes of these waves not only provides understanding about the internal structure of the rock, but also gives information about the valuable hydrocarbons present inside the earth crust. Since the inception of the theory of wave propagation in a porous medium by Biot (1956a, b), many researchers have attempted the study of reflection and transmission of wave at the interface between two dissimilar media. Geertsma and Smith (1961) studied the reflection and absorption of wave at an interface between two different fluid saturated porous solids. They studied the effect of Biot's slow wave on reflection and absorption of wave incident normally at the interface. Deresiewicz and Rice (1962) studied the phenomenon of reflection from a plane, traction free boundary in a dissipative medium, for each of three body waves predicted by Biot's theory. They observed no mode conversion of a wave incident normally and at $45^{\circ}$ at the interface. They obtained expressions for the phase velocities, attenuation coefficients, angle of reflection and the amplitude ratios of reflected waves. Deresiewicz and Levy (1967) calculated reflection and transmission coefficients for the propagation of stress-waves through the system of porous layers separating two porous half-spaces. For simplicity, they confined their attention to the normal incidence, where no mode conversion was observed during reflection. Hajra and Mukhopadhyay (1982) extended the problem to more general situation. They studied reflection and transmission of waves incident obliquely at an interface between elastic half-space and a porous half-space saturated by a fluid. They employed boundary conditions as suggested by Deresiewicz and Skalk (1963). Dutta and Ode (1983) studied seismic reflections at a gaswater boundary in a porous sand reservoir. They considered waves incident obliquely at the interface of two-fluid filled porous rocks. They considered inhomogeneous waves due to dissipative nature of the medium and introduced interference fluxes of energy between various types of waves. Notable study on reflection and refraction of an inhomogeneous wave is given by (Borcherdt 1977, 1982). Vashisth et al. (1991) studied reflection and transmission at a loosely bounded interface between an elastic solid and a liquid filled porous solid. They observed that energy of incident wave dissipates due to loosely bounded interface. Santos et al. (1992) analyzed the effect of the inclusion of frequency correction parameter in Biot's equations for the calculation of reflection and transmission coefficients at plane interfaces. Plane interfaces considered in their study are a fluid-Biot medium 
interface and an interface within a Biot's medium defined by a change in permeability or in the saturant fluid. Sharma and Saini (1992) defined a pore connectivity parameter to classify the connection between the interstices of two media. They studied the effect of variation in pore connectivity parameter on the amplitude ratios and energy ratios of an incident wave. Sharma (2008) further elaborated this problem and derived the set of boundary conditions to represent the partial connection of surface pores at the porous-porous interface. They showed that at plane interface, the imperfection in welded bonding is represented by tangential slipping and results in dissipation of strain energy. Sharma (2004) studied reflection/refraction process to calculate energy distribution in a general anisotropic poroelastic half-space in contact with a fluid half-space.

Reflection and refraction of plane harmonic waves at boundary of porous media saturated by two immiscible fluids were given by (Tomar and Arora (2006); Arora and Tomar (2007) and Yeh et al. (2010)). Kumar and Sharma (2013) studied reflection and transmission of plane harmonic waves at the plane interface between two dissimilar poroelastic solids saturated with two immiscible viscous fluids. Due to the presence of viscous porefluids, the medium in their study was considered to be dissipative in nature. Inhomogeneity of waves was described by specifying different directions of propagation and attenuation. They also used a parameter to define connections among the surface pores of two media at interface.

Starting with the classical work of Geertsma and Smith (1961), the study of reflection and transmis- saturated by two immiscible viscous fluids. Model equations developed by Arora et al. (2016) for wave propagation in a composite solid matrix saturated by two fluids are employed for the study. When an inhomogeneous wave strikes the interface, it scatters into six reflected and six transmitted waves. Among these reflected/transmitted waves, four are dilatational in nature while two are rotational. The expression for Umov-Poynting vector is derived for the system to obtain energy flux vector. Appropriate boundary conditions are obtained using continuity of energy flux vector at the interface and by introducing pore connection parameter. Reflection/transmission and energy coefficients are obtained and their dependency upon angle of incidence at three distinct values of one of the solid fraction and pore connecting parameter is studied for a porous matrix composed of sandstone and ice particles, saturated with oil and water. Due to inhomogeneous nature of waves the interference energy and the orthodox energy fluxes are also given consideration to verify energy balance at the interface.

\section{Field equations}

We consider a porous matrix composed of two solids, and saturated with two immiscible fluids. The differential equations governing deformation of such a medium, in absence of body forces are given by Arora et al. (2016)

$$
L U=N,
$$

where
$L=\left[\begin{array}{cc}\left(\rho_{s_{1}} \theta_{s_{1}}-\mathbb{A}\right) D^{2}-\mathbb{R} D & \mathbb{B} \\ \mathbb{B} & \left(\rho_{1} \theta_{1}-\mathbb{E}\right) D^{2}-2 R_{11} D \\ \mathbb{C} & -4 A_{12} D^{2} \\ \mathbb{D} & \mathbb{B}\end{array}\right.$

sion of waves still continues due to its increasing applicability and academic interest. Recently, the study conducted by Zhao and Shen (2015) and Deckers et al. (2016) is worth mentioning in this regard. The present work studies the effect of pore connection on the reflection and transmission of an inhomogeneous wave striking at the interface of two dissimilar porous media. Both the media are assumed to be composed of two distinct solids and

$$
\begin{gathered}
\mathbb{C} \\
-4 A_{12} D^{2} \\
\left.\begin{array}{c}
\left(\rho_{2} \theta_{2}-\mathbb{F}\right) D^{2}-2 R_{22} D \\
\mathbb{C}
\end{array}\right], \\
U=\left[\begin{array}{c}
\boldsymbol{u}_{s_{1}} \\
\boldsymbol{u}_{1} \\
\boldsymbol{u}_{2} \\
\boldsymbol{u}_{s_{2}}
\end{array}\right], \quad N=\left[\begin{array}{c}
\nabla \cdot \sigma^{s_{1}} \\
\nabla \sigma^{1} \\
\nabla \sigma^{2} \\
\nabla \cdot \sigma^{s_{2}}
\end{array}\right] .
\end{gathered}
$$

The subscripts $s_{1}, s_{2}$ and 1,2 are used to denote two solid phases and two fluid phases, respectively. Components $\rho_{\alpha}, \theta_{\alpha}$ and $\boldsymbol{u}_{\alpha}(\alpha=$ $\left.s_{1}, s_{2}, 1,2\right)$ denote, respectively the mass density, 
volume fraction and displacement field of each phase. The symbol $\sigma^{\alpha}$ corresponds to partial stress on each phase and $D \equiv \partial / \partial t$ represents the temporal derivative operator. Coefficients $R_{11}$ and $R_{22}$ symbolize the viscous drag between fluid particles and solid particles due to first and second fluid, respectively. Inertial drags of first solid with first and second fluid are respectively represented by $A_{11}$ and $A_{22}$. Similarly, $B_{11}$ and $B_{22}$ denote inertial coupling of second solid with both of the fluids. Inertial coupling connecting fluid phases is specified by $A_{12}$. The expressions of viscous and inertial drag coefficients are given in Appendix 1. The expressions of other elements appearing in the matrix $L$ are given as follows: where the scalar potentials $\phi_{\alpha}$ and vector potential $\psi_{\alpha}$, respectively, correspond to dilatation and distortion in the medium. For propagation of plane wave we consider the following time harmonic solution.

$$
\left\{\phi_{\alpha}, \psi_{\alpha}\right\}=\left\{\bar{\phi}_{\alpha}, \bar{\psi}_{\alpha}\right\} \exp ^{-\imath \omega t}
$$

where $\bar{\phi}_{\alpha}$ and $\overline{\boldsymbol{\psi}}_{\alpha}$ represent, respectively, the polarizations for the motion of particles of each phase in composite matrix. Using equations (3) and (4), the general field equations given by (1) split into the following equations.

$$
\Lambda_{\mathfrak{f}} \Gamma_{\mathfrak{f}}=\mathcal{O}, \quad(\mathfrak{f}=1,2)
$$

where

$$
\begin{gathered}
\Lambda_{1}=\left(\begin{array}{cccc}
\bar{a}_{11} Y+\eta_{00} & a_{12} Y+\eta_{01} & a_{13} Y+\eta_{02} & a_{14} Y+\eta_{03} \\
a_{12} Y+\eta_{01} & a_{22} Y+\eta_{11} & a_{23} Y+\eta_{12} & a_{24} Y+\eta_{01} \\
a_{13} Y+\eta_{02} & a_{23} Y+\eta_{12} & a_{33} Y+\eta_{23} & a_{34} Y+\eta_{02} \\
a_{14} Y+\eta_{03} & a_{24} Y+\eta_{01} & a_{34} Y+\eta_{02} & \bar{a}_{44} Y+\eta_{44}
\end{array}\right), \quad Y=\frac{\nabla^{2}}{\omega^{2}}, \\
\Lambda_{2}=\left(\begin{array}{cccc}
G_{s_{1}} Y+\eta_{00} & \eta_{01} & \eta_{02} & \eta_{03} \\
\eta_{01} & \eta_{11} & \eta_{12} & \eta_{01} \\
\eta_{02} & \eta_{12} & \eta_{23} & \eta_{02} \\
\eta_{03} & \eta_{01} & \eta_{02} & G_{s_{2}} Y+\eta_{44}
\end{array}\right), \quad \Gamma_{1}=\left(\begin{array}{c}
\bar{\phi}_{s_{1}} \\
\bar{\phi}_{1} \\
\bar{\phi}_{2} \\
\bar{\phi}_{s_{2}}
\end{array}\right), \Gamma_{2}=\left(\begin{array}{c}
\overline{\boldsymbol{\psi}}_{s_{1}} \\
\overline{\boldsymbol{\psi}}_{2} \\
\overline{\boldsymbol{\psi}}_{s_{2}}
\end{array}\right),
\end{gathered}
$$

$$
\begin{aligned}
& \mathbb{A}=A_{11}+2 A_{12}+A_{22}, \\
& \mathbb{B}=\left(A_{11}+2 A_{12}+B_{11}\right) D^{2}+R_{11} D, \\
& \mathbb{C}=\left(A_{22}+2 A_{12}+B_{22}\right) D^{2}+R_{22} D, \\
& \mathbb{D}=-\left(B_{11}+2 A_{12}+B_{22}\right) D^{2}, \\
& \mathbb{E}=2\left(A_{11}+B_{11}\right), \mathbb{F}=2\left(A_{22}+B_{22}\right), \\
& \mathbb{R}=R_{11}+R_{22} .
\end{aligned}
$$

Constitutive relations, expressing stress on each phase may be given by

$$
[\sigma]=[\mathcal{L}][\epsilon],
$$

where $[\sigma]$ and $[\epsilon]$ are column matrices of order fourteen, expressing respectively the components of stress and strain tensors. The square matrix $[\mathcal{L}]$ is a symmetric matrix of order fourteen. The expressions of the elements of these matrices are given in Appendix 1.

Using Helmholtz resolution of vectors the displacement vector $\mathbf{u}_{\alpha}$ may be represented as

$$
\mathbf{u}_{\alpha}=\nabla \phi_{\alpha}+\nabla \times \boldsymbol{\psi}_{\alpha}, \nabla \cdot \boldsymbol{\psi}_{\alpha}=0,
$$

$\mathcal{O}$ is a null matrix. Also we have

$$
\begin{aligned}
& \bar{a}_{11}=a_{11}+\frac{4}{3} G_{s_{1}}, \bar{a}_{44}=a_{44}+\frac{4}{3} G_{s_{2}}, \\
& \eta_{00}=\rho_{s_{1}} \theta_{s_{1}}-\mathbb{A}-\frac{\iota}{\omega} \mathbb{R} \\
& \eta_{44}=\rho_{s_{2}} \theta_{s_{2}}-\mathbb{A}-\frac{\iota}{\omega} \mathbb{R} \\
& \eta_{03}=-\left(B_{11}+B_{22}+2 A_{12}\right) \\
& \eta_{01}=A_{11}+2 A_{12}+B_{11}+\frac{\iota}{\omega} R_{11}, \\
& \eta_{02}=A_{22}+2 A_{12}+B_{22}+\frac{\iota}{\omega} R_{22}, \\
& \eta_{11}=\rho_{1} \theta_{1}-2\left(A_{11}+B_{11}\right)-2 \frac{\iota}{\omega} R_{11} \\
& \eta_{12}=-4 A_{12}, \\
& \eta_{23}=\rho_{2} \theta_{2}-2\left(A_{22}+B_{22}\right)-2 \frac{\iota}{\omega} R_{22} .
\end{aligned}
$$

This represents the system of four homogeneous equations in scalar and vector potentials for $\mathfrak{f}=1$ and $\mathfrak{f}=2$, respectively. Condition for the non-trivial solution of this system yields following partial differential equations 


$$
\begin{aligned}
& \left(A Y^{4}+B Y^{3}+C Y^{2}+D Y+E\right) \bar{\phi}_{s_{1}}=0 \\
& \left(G Y^{2}+H Y+I\right) \overline{\boldsymbol{\psi}}_{s_{1}}=0
\end{aligned}
$$

The expressions of coefficients appearing in above differential equations are very large and not produced here to avoid undue length of the article.

General solution of (6) may be written as:

$$
\left(\bar{\phi}_{s_{1}}, \bar{\psi}_{s_{1}}\right)=\left(\sum_{\beta=1}^{4} \phi^{\beta}, \quad \sum_{l=5}^{6} \boldsymbol{\psi}^{l}\right)
$$

where $\phi^{\beta}$ and $\boldsymbol{\psi}^{l}$ satisfy the Helmholtz equation

$$
\left(\nabla^{2}+k_{\beta}^{2}\right) \phi^{\beta}=0, \quad\left(\nabla^{2}+k_{l}^{2}\right) \boldsymbol{\psi}^{l}=0 .
$$

This indicates the existence of four dilatational waves and two shear waves in the medium. The symbols $k_{\beta}$ and $k_{l}$ correspond to complex wavenumbers of dilatational and shear waves, respectively. The propagation velocities of dilatational waves can be found by solving the equation $E v^{8}-D v^{6}+$ $C v^{4}-B v^{2}+A=0$. Out of the eight roots of this equation, the four roots with positive real part give the velocities of four dilatational waves travelling in the medium. These four dilatational waves along with their velocities may be represented by the pair $\left(v_{\beta}, P \beta\right)$, where $(\beta=1,2,3,4)$. Similarly, the velocities of the transverse waves can be found by solving the equation $I v^{2}-H v+G=0$. Two roots of this equation give the velocities of two transverse waves travelling in the medium. These two waves along with their velocities can be represented by the pairs $\left(v_{5}, S 1\right)$ and $\left(v_{6}, S 2\right)$.

Using second, third and fourth equations from the set of equations represented by equation (5), we find the following relations.

$$
\begin{aligned}
& \left(\bar{\phi}_{1}: \bar{\phi}_{2}: \bar{\phi}_{s_{2}}: \bar{\phi}_{s_{1}}\right)=\kappa_{1}: \kappa_{2}: \kappa_{3}: \kappa_{4} \\
& \left(\overline{\boldsymbol{\psi}}_{1}: \overline{\boldsymbol{\psi}}_{2}: \overline{\boldsymbol{\psi}}_{s_{2}}: \overline{\boldsymbol{\psi}}_{s_{1}}\right)=\epsilon_{1}: \epsilon_{2}: \epsilon_{3}: \epsilon_{4}
\end{aligned}
$$

where $\kappa_{\beta}$ and $\epsilon_{\beta}$ are the coupling coefficients. The expressions of these coefficients are given in Appendix 2.

Substituting equations (9-10) into (7) and using (8), we obtain the following coupled relations.

$$
\bar{\phi}_{\alpha}=\lambda_{\alpha}^{\beta} \phi^{\beta}, \quad \overline{\boldsymbol{\psi}}_{\alpha}=\lambda_{\alpha}^{l} \boldsymbol{\psi}^{l},
$$

where summation convention is used over the repeated index. The expressions of coupling relations are given in Appendix 2.
Due to dissipation in the medium, the waves become inhomogeneous in nature (Borcherdt 1982). In an inhomogeneous wave, the direction of attenuation is always different than the direction of propagation. The angle at which attenuation vector is inclined to its propagation vector describes the inhomogeneity of a wave. Expressions of propagation and attenuation vectors in terms of its slowness vector are defined by Vlastislav and Ivan (2005); Kumar and Sharma (2013).

$$
\begin{aligned}
& \mathbf{P}=\omega\left\{\Re\left(s_{x}\right) \hat{x}-\Re\left(s_{z}\right) \hat{z}\right\}, \\
& \mathbf{A}=\omega\left\{-\Im\left(s_{x}\right) \hat{x}+\Im\left(s_{z}\right) \hat{z}\right\},
\end{aligned}
$$

where $s_{x}$ and $s_{z}$ respectively represent horizontal and vertical slowness of a wave and symbols $\Re$ and $\Im$ signify the real and imaginary parts of a complex quantity. We assume that the attenuation vector $\mathbf{A}$ is inclined to the propagation vector $\mathbf{P}$ at an angle $\gamma$, such that $0<\gamma<\pi / 2$. The expressions for phase speed $V$ and maximum attenuation $A$ of an inhomogeneous wave are given by Sharma (2005)

$$
\begin{aligned}
|V|= & |P|^{-1}=\left[\frac { 1 } { 2 } \left\{\Re\left(v_{m}{ }^{-2}\right)\right.\right. \\
& \left.\left.+\sqrt{\left\{\Re\left(v_{m}^{-2}\right)\right\}^{2}+\left\{\Im\left(v_{m}^{-2}\right)\right\}^{2} \sec ^{2} \gamma}\right\}\right]^{\frac{-1}{2}}, \\
|A|= & {\left[\frac { 1 } { 2 } \left\{-\Re\left(v_{m}{ }^{-2}\right)\right.\right.} \\
& \left.\left.+\sqrt{\left\{\Re\left(v_{m}^{-2}\right)\right\}^{2}+\left\{\Im\left(v_{m}^{-2}\right)\right\}^{2} \sec ^{2} \gamma}\right\}\right]^{\frac{1}{2}} \\
& m=1,2, \ldots, 6 .
\end{aligned}
$$

It is obvious from equations (13) and (14) that attenuation vector of the wave cannot be perpendicular to propagation vector.

\section{Energy flux and boundary conditions}

Our aim is to study the effect of pore connectivity among the pores of distinct media on reflection amplitudes of an inhomogeneous wave. We consider two distinct half spaces $\Omega$ and $\Omega^{\prime}$, composed of two solids and saturated by two immiscible fluids. In a rectangular co-ordinate system $O x y z$, the halfspace $\Omega$ occupies the region $(-\infty \leq z<0)$ and $\Omega^{\prime}$ occupies the region $(0<z \leq \infty)$. When a beam of plane elastic inhomogeneous wave after propagating through $\Omega$ impinges on the interface $z=0$, it 
scatters into 12 distinct reflected and transmitted waves.

For wave propagation along the interface, it is necessary that the energy flux vector balances at the interface. This condition also helps in framing out the required boundary conditions for the system. Therefore, we derive an expression for energy flux vector for our medium. Taking product of equation of motion of each phase with conjugate of its respective velocity, equation of motion (1) becomes

$$
\mathbf{v}^{* T}\left(\mathcal{K} D^{2}+\mathcal{R} D\right) U(x, t)=\mathbf{v}^{* T} N[U(x, t)],
$$

where $\left(\mathcal{K} D^{2}+\mathcal{R} D\right)=L$ and $\mathbf{v}=\left(\mathbf{v}^{s_{1}} \mathbf{v}^{1} \mathbf{v}^{2} \mathbf{v}^{s_{2}}\right)^{T}$. The symbol $*$ represents the complex conjugate of the matrix and $\mathbf{v}^{\alpha}$ represents the velocity vector of the phase $\alpha$. In time harmonic field $\exp \{\iota \omega t\}$, equation (15) becomes

$$
-\omega^{2} \mathbf{v}^{* T} \mathcal{M U}(x, \omega)=\mathbf{v}^{* T} N[U(x, \omega)],
$$

where $\mathcal{M}=\left(\mathcal{K}-\frac{\iota}{\omega} \mathcal{R}\right)$. Invoking the stress strain relations given by (2), the right hand side of equation (16) may be expressed as:

$$
\begin{aligned}
\mathbf{v}^{* T} N[U]= & \nabla \cdot\left[\sigma_{i j}^{s_{1}}(U)\left(v_{j}^{s_{1}}\right)^{*}+\sigma^{1}(U)\left(v_{i}^{1}\right)^{*}\right. \\
& \left.+\sigma^{2}(U)\left(v_{i}^{2}\right)^{*}+\sigma_{i j}^{s_{2}}(U)\left(v_{j}^{s_{2}}\right)^{*}\right] \\
& -\left(\sigma_{i j}^{s_{1}}(U) \epsilon_{i j}\left(v^{s_{1}}\right)^{*}+\sigma^{1}(U) \nabla \cdot\left(v^{1}\right)^{*}\right. \\
& \left.+\sigma^{2}(U) \nabla \cdot\left(v^{2}\right)^{*}+\sigma_{i j}^{s_{2}}(U) \epsilon_{i j}\left(v^{s_{2}}\right)^{*}\right), \\
& x \leq i, j \leq z .
\end{aligned}
$$

Using above relation in equation (16) and integrating over the volume $\mathbf{V}$, we obtain

$$
\begin{gathered}
\iota \omega \int_{\mathbf{V}}\left(\epsilon^{* T}(U) \mathcal{L} \epsilon(U)-\mathbf{v}^{* T} \mathcal{M} \mathbf{v}\right) d \mathbf{V} \\
=-\int_{\mathbf{V}} \nabla \cdot\left(\sigma_{i j}^{s_{1}}\left(\mathrm{v}_{j}^{s_{1}}\right)^{*}+\sigma^{1}\left(\mathrm{v}_{i}^{1}\right)^{*}\right. \\
\left.\quad+\sigma^{2}\left(\mathrm{v}_{i}^{2}\right)^{*}+\sigma_{i j}^{s_{2}}\left(\mathrm{v}_{j}^{s_{2}}\right)^{*}\right) d \mathbf{V} .
\end{gathered}
$$

For partially sealed pores, discharge at interface is proportional to effective stress (Biot 1962; Sharma and Saini 1992; Sharma 2009). Effective stress corresponds to difference between normal stress of composite matrix and fluid pressure. Therefore, we can re-write above equation as follows:

$$
\begin{aligned}
\iota \omega & \int_{\mathbf{V}}\left(\epsilon^{* T}(U) \mathcal{L} \epsilon(U)-\mathbf{v}^{*^{T}} \mathcal{M} \mathbf{v}\right) d \mathbf{V} \\
= & -\int_{\mathbf{V}} \nabla \cdot\left(\sigma_{i j}^{s_{1}}\left(v_{j}^{s_{1}}\right)^{*}+\Gamma^{1}\left(w_{i}^{1}\right)^{*}+\Gamma^{2}\left(w_{i}^{2}\right)^{*}\right. \\
& \left.+\sigma_{i j}^{s_{2}}\left(v_{j}^{s_{2}}\right)^{*}\right) d \mathbf{V} \\
= & -\int_{\mathbf{S}}\left(\sigma_{i j}^{s_{1}}\left(v_{j}^{s_{1}}\right)^{*}+\Gamma^{1}\left(w_{i}^{1}\right)^{*}+\Gamma^{2}\left(w_{i}^{2}\right)^{*}\right. \\
& \left.+\sigma_{i j}^{s_{2}}\left(v_{j}^{s_{2}}\right)^{*}\right) \cdot \hat{a} d \mathbf{S} .
\end{aligned}
$$

where $\Gamma^{\mathfrak{f}}=\sigma^{\mathfrak{f}}-\theta_{\mathfrak{f}} \sigma, w_{i}^{\mathfrak{f}}=\left(v_{i}^{\mathfrak{f}}-\theta_{s_{1}} v_{i}^{s_{1}}-\theta_{s_{2}} v_{i}^{s_{2}}\right)$ and $\sigma$ is the sum of normal stresses of each phase in composite matrix. The symbols $\Gamma^{f}$ and $w_{i}^{f}$ correspond to effective stress at interface and normal component of drainage velocity of fluids, respectively and the symbol $\hat{a}$ is used for unit normal vector on the surface $\mathbf{S}$.

Equation (18) represents the energy balance equation for the system. To understand various terms in this equation we consider the following time-averaged relations (Rubino et al. 2006; Carcione 2014).

$$
\begin{aligned}
\Re\left(\epsilon^{* T} \mathcal{L} \epsilon\right) & =2\left\langle\Re\left(\epsilon^{T}\right) \Re(\mathcal{L}) \Re(\epsilon)\right\rangle, \\
\Im\left(\epsilon^{T} \mathcal{L} \epsilon\right) & =2\left\langle\Re\left(\epsilon^{T}\right) \Im(\mathcal{L}) \Re(\epsilon)\right\rangle, \\
\Re\left(\mathbf{v}^{* \mathbf{T}} \mathcal{M} \mathbf{v}\right) & =2\left\langle\Re\left(\mathbf{v}^{T}\right) \Re(\mathcal{M}) \Re(\mathbf{v})\right\rangle \\
& =2\left\langle\Re\left(\mathbf{v}^{T}\right) \mathcal{K} \Re(\mathbf{v})\right\rangle, \\
\Im\left(\mathbf{v}^{* \mathbf{T}} \mathcal{M} \mathbf{v}\right) & =\left\langle\Re\left(\mathbf{v}^{\mathbf{T}}\right) \Im(\mathcal{M}) \Re(\mathbf{v})\right\rangle \\
& =2\left\langle\Re\left(\mathbf{v}^{\mathbf{T}}\right) \mathcal{R} \Re(\mathbf{v})\right\rangle,
\end{aligned}
$$

where $\langle\cdot\rangle$ represents time average of a quantity over a cycle of period $2 \pi / \omega$. Taking into account equations (19-21) we may write equation (18) as:

$\iota \omega \int_{\mathbf{V}} 2(\mathcal{W}-\mathcal{T}) d \mathbf{V}-\int_{\mathbf{V}}\left(D_{\mathcal{W}}+D_{\mathcal{T}}\right) d \mathbf{V}=\int_{\mathbf{S}} \mathcal{P} \cdot \hat{a} d \mathbf{S}$,

where $\mathcal{P}$ is a complex Umov-Poynting vector of a medium. In component notation, this vector can be written as:

$$
\begin{aligned}
\mathcal{P}_{k}= & -\frac{1}{2}\left(\sigma_{k j}^{s_{1}}\left(v_{j}^{s_{1}}\right)^{*}+\Gamma^{1}\left(w_{k}^{1}\right)^{*}\right. \\
& \left.+\Gamma^{2}\left(w_{k}^{2}\right)^{*}+\sigma_{k j}^{s_{2}}\left(v_{j}^{s_{2}}\right)^{*}\right),
\end{aligned}
$$

The expressions of $\mathcal{W}$ and $\mathcal{T}$ are given as follows:

$$
\begin{aligned}
& \mathcal{W}=\frac{1}{2}\left\langle\Re\left(\epsilon^{T}\right) \Re(\mathcal{L}) \Re(\epsilon)\right\rangle, \\
& \mathcal{T}=\frac{1}{2}\left\langle\Re\left(\mathbf{v}^{T}\right) \mathcal{K} \Re(\mathbf{v})\right\rangle,
\end{aligned}
$$




$$
\begin{aligned}
& D_{\mathcal{W}}=\omega\left\langle\Re\left(\epsilon^{T}\right) \Im(\mathcal{L}) \Re(\epsilon)\right\rangle, \\
& D_{\mathcal{T}}=-\omega\left\langle\Re\left(\mathbf{v}^{T}\right) \mathcal{R} \Re(\mathbf{v})\right\rangle,
\end{aligned}
$$

where $\mathcal{W}$ and $\mathcal{T}$ in equation (24) are analogous to potential and kinetic energy density, respectively. Whereas $D_{\mathcal{W}}$ and $D_{\mathcal{T}}$ correspond to rate of dissipations in corresponding energy densities. by

The real part of Umov-Poynting vector is given

$$
\begin{aligned}
\Re\left(\mathcal{P}_{k}\right)= & -\left(\Re\left(\sigma_{k j}^{s_{1}}\right) \Re\left(v_{j}^{s_{1}}\right)+\Re\left(\Gamma^{1}\right) \Re\left(w_{k}^{1}\right)\right. \\
& \left.+\Re\left(\Gamma^{2}\right) \Re\left(w_{k}^{2}\right)+\Re\left(\sigma_{k j}^{s_{2}}\right) \Re\left(v_{j}^{s_{2}}\right)\right) .
\end{aligned}
$$

This represents the magnitude and direction of the time-averaged power flow (Rubino et al. 2006; Carcione 2014). The time-averaged energy fluxes along normal to the interface in both the half-spaces are given by

$$
\left(F, F^{\prime}\right)=\frac{\omega}{2 \pi} \int_{0}^{2 \pi / \omega}\left(\Re\left(\mathcal{P}_{z}\right), \Re\left(\mathcal{P}_{z}\right)^{\prime}\right) \cdot \hat{z} d t .
$$

Continuity of energy flux at interface gives 10 boundary conditions which are given in following three sets

$$
\begin{aligned}
& \sigma_{z z}^{s_{\mathfrak{f}}}=\sigma_{z z}^{s_{\mathfrak{f}}}, \quad \sigma_{x z}^{s_{\mathfrak{f}}}=\sigma_{x z}^{s_{\mathfrak{f}}},
\end{aligned}
$$

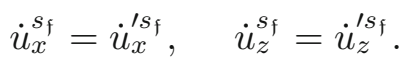

$$
\begin{aligned}
& \frac{\Gamma^{1}}{w_{z}^{1^{\prime}}}=\frac{\Gamma^{1 \prime}}{w_{z}^{1}}, \quad \frac{\Gamma^{2}}{w_{z}^{2^{\prime}}}=\frac{\Gamma^{2 \prime}}{w_{z}^{2}} .
\end{aligned}
$$

We aim to study a situation where the pores of two media are partially closed. In a realistic situation the pores at the interface of two media may not be fully connected. The pores at the interface of two distinct media may be closed or sealed due to non-connectivity of pores or due to some other weathering conditions like deposition of sand or solid grains at interstices (Arora and Tomar 2007) or the difference of porosities in both the media. The parameter $\varsigma \in[0,1]$ is introduced to describe the connectivity of pores. The zero value of $\varsigma$ corresponds to the totally sealed pores, while $\varsigma=1$ corresponds to totally open pores. For $\varsigma \in(0,1)$, the pores are partially connected. Figure 1 depicts the situation of connectivity of pores of two porous media at interface. This parameter may be defined mathematically as:

$$
\varsigma=\frac{\operatorname{Min}\left(f, f^{\prime}\right)}{\operatorname{Max}\left(f, f^{\prime}\right)} \ell,
$$

where $f$ and $f^{\prime}$ symbolize porosities of two media. The porosity of composite porous medium is assumed to be distributed uniformly and may be considered as the union of porosities of both solids. The parameter $\varsigma$ depends upon porosities of the media. The other factors like weathering are depicted by the parameter $\ell$. As the porosity of $\Omega$ approaches towards the porosity of $\Omega^{\prime}$, the parameter $\varsigma$ automatically approaches towards 1 for $\ell=1$. The significance of parameter $\ell$ lies in the fact that even in case of equal porosities of two media, there can be a disconnection among the pores. We introduce the connecting parameter $\varsigma$ in the boundary conditions (equation 30) to signify the extent of

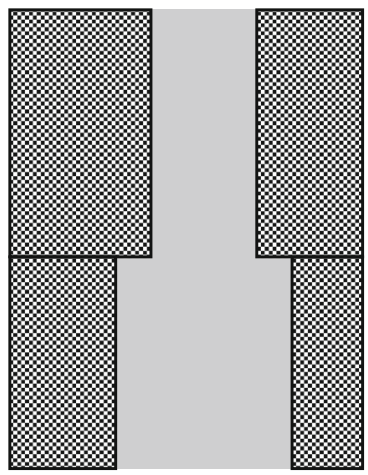

(a) $(\varsigma=1)$

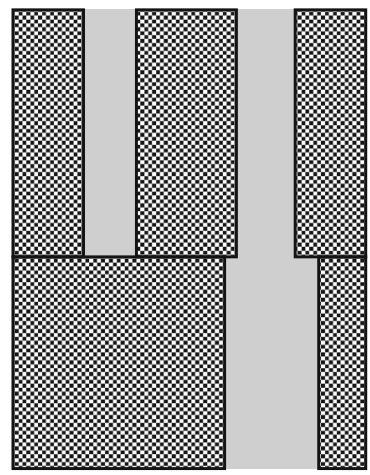

(b) $(\varsigma$ in $[0,1])$

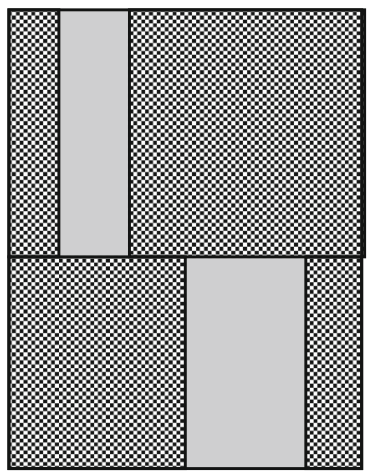

(c) $(\varsigma=0)$

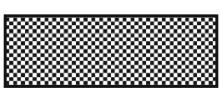

Solid phase

Fluid phase

Figure 1. Pore connectivity at the interface of two media with the varying values of $\varsigma$. 
connection between the media. This gives us the following four boundary conditions

$$
\begin{aligned}
& \varsigma \frac{\Gamma^{1}}{w_{z}^{1^{\prime}}}=T_{1}(1-\varsigma), \quad \varsigma \frac{\Gamma^{2}}{w_{z}^{2^{\prime}}}=T_{2}(1-\varsigma) \\
& \varsigma \frac{\Gamma^{1^{\prime}}}{w_{z}^{1}}=T_{1}(1-\varsigma), \quad \varsigma \frac{\Gamma^{2^{\prime}}}{w_{z}^{2}}=T_{2}(1-\varsigma),
\end{aligned}
$$

where $T_{1}$ and $T_{2}$ signify the surface flow impedance for both fluids (Denneman et al. 2002). Equations (28), (29) and (32) give the boundary conditions for partially sealed pores at interface, for $0 \leq \varsigma \leq 1$.

\section{Reflection and transmission}

Let a beam of plane elastic inhomogeneous dilatational wave with propagation vector $\mathbf{P}_{o}$, attenuation vector $\mathbf{A}_{\mathbf{o}}$ and attenuation angle $\gamma_{o}$ after travelling through $\Omega$, strikes the interface $Z=0$ at an angle $\theta_{o}$ with the normal. This gives rise to 12 reflected and transmitted waves. Four $P$ and two $S V$ waves are reflected back in the medium $\Omega$, while four $P$ and two $S V$ waves are transmitted into $\Omega^{\prime}$. Reflected and transmitted waves are also inhomogeneous in nature and have different directions of propagation and attenuation vectors as shown in figure 2 .

The propagation vectors of six waves in $\Omega$ are denoted by $\left(\mathbf{P}_{m}, m=1,2, \ldots, 6\right)$ and these vectors are directed at an angle $\theta_{m}$ with the normal. Attenuation vectors of these waves are depicted by $A_{m}$ and are inclined at an angle $\gamma_{m}$ with their propagation vectors. Similarly in $\Omega^{\prime}$, four $P$ and two $S$ waves have corresponding propagation and attenuation vectors given by $\left(\mathbf{P}_{m}^{\prime}, \mathbf{A}_{m}^{\prime}, \gamma_{m}^{\prime}\right)$.

Displacement vectors of solid and fluid particles along $x$ and $z$ directions in $\Omega$ and $\Omega^{\prime}$ are given by

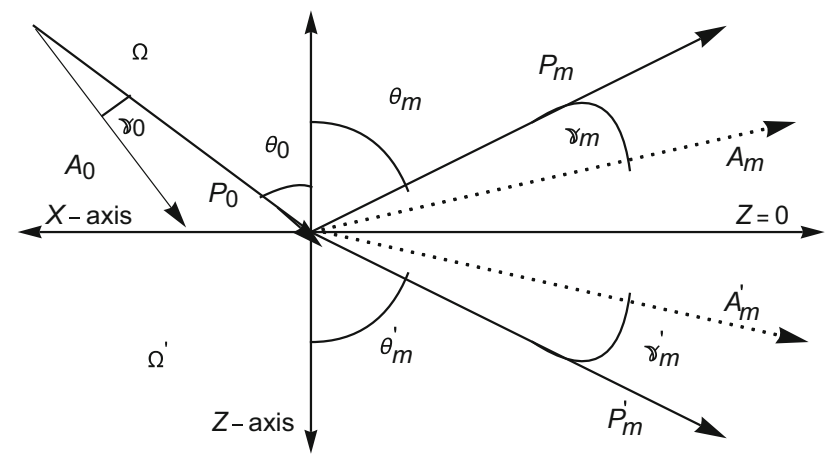

Figure 2. Geometrical representation of reflection and transmission of waves at interface of composite porous media.

$$
\begin{aligned}
\mathbf{u}_{\alpha}= & \left(u_{\alpha x}, 0, u_{\alpha z}\right)=\left(\mathbf{u}_{\alpha}\right)_{\mathrm{Inc}}+\left(\mathbf{u}_{\alpha}\right)_{\text {Refl }} \\
= & \lambda_{\alpha}^{\beta} \nabla \phi^{o}+\sum_{\beta=1}^{4} \lambda_{\alpha}^{\beta} \nabla \phi^{\beta}+\sum_{l=5}^{6} \lambda_{\alpha}^{l} \nabla \times \boldsymbol{\psi}^{l},(3) \\
\mathbf{u}_{\alpha}^{\prime}= & \left(u_{\alpha x}{ }^{\prime}, 0, u_{\alpha z}{ }^{\prime}\right)=\left(\mathbf{u}_{\alpha}\right)_{\mathrm{Refr}}=\sum_{\beta=1}^{4} \lambda_{\alpha}^{\beta^{\prime}} \nabla \phi^{\beta^{\prime}} \\
& +\sum_{l=5}^{6} \lambda_{\alpha}^{l^{\prime}} \nabla \times \boldsymbol{\psi}^{l^{\prime}} .
\end{aligned}
$$

\subsection{Amplitude ratios}

Now we proceed to find the amplitude ratio of each reflected and transmitted wave to that of incident wave. The horizontal component of slowness vector for the incident wave may be expressed as:

$$
s_{x}=\frac{\left|\mathbf{P}_{o}\right|}{\omega} \sin \theta_{o}-\iota \frac{\left|\mathbf{A}_{o}\right|}{\omega} \sin \left(\theta_{o}-\gamma_{o}\right) .
$$

Since by generalized Snell's law horizontal components of slowness vector of reflected/refracted waves and incident wave must be equal (Borcherdt 1982), therefore

$$
\begin{aligned}
\left|\mathbf{P}_{o}\right| \sin \theta_{o} & =\left|\mathbf{P}_{m}\right| \sin \theta_{m}=\left|\mathbf{P}_{m}^{\prime}\right| \sin \theta_{m}^{\prime} \\
\left|\mathbf{A}_{o}\right| \sin \left(\theta_{o}-\gamma_{o}\right) & =\left|\mathbf{A}_{m}\right| \sin \left(\theta_{m}-\gamma_{m}\right) \\
& =\left|\mathbf{A}_{m}^{\prime}\right| \sin \left(\theta_{m}^{\prime}-\gamma_{m}^{\prime}\right)
\end{aligned}
$$

All waves are distinguished by the vertical component of their slowness vector. For brevity, the components of slowness vector may be called vertical slowness and horizontal slowness. For wave velocity $v$, vertical slowness of a wave is connected to its horizontal slowness by the following relation.

$$
s_{z}=\sqrt{\left(v^{-2}-s_{x}^{2}\right)} .
$$

The principal value is chosen to ensure that $\Im\left(s_{z}\right) \geq 0$. This confirms that the corresponding wave decays in positive $z$ direction.

The expressions for potentials corresponding to various dilatational and shear waves in both the media are given by

For incidence wave:

$$
\phi^{o}=Z_{o} \exp \left\{\iota \omega\left(s_{x} x+s_{o z} z-t\right)\right\} .
$$

For reflected and transmitted waves:

$$
\begin{gathered}
\phi^{m}=Z_{m} \exp \left\{\iota \omega\left(s_{x} x-s_{m z} z-t\right)\right\}, \\
\phi^{m \prime}=Z_{m}^{\prime} \exp \left\{\iota \omega\left(s_{x} x+s_{m z}^{\prime} z-t\right)\right\} ; \\
(m=1,2,3, \ldots, 6)
\end{gathered}
$$


where $Z_{o}, Z_{m}$ and $Z_{m}^{\prime}$ are amplitudes of incident, reflected and transmitted waves, respectively. The symbols $\phi^{5}$ and $\phi^{6}$, respectively represent $y$ component of vectors $\boldsymbol{\psi}^{5}$ and $\boldsymbol{\psi}^{6}$. Invoking Snell's law and substituting the expressions of potentials given by equations (37-39) into the equations (33) and (34), the 12 boundary conditions (equations 28, 29 and 32), yield 12 non-homogeneous linear equations with 12 unknowns. These equations can be written in matrix form in the following way.

$$
\mathcal{B Z}=\mathcal{C}
$$

where $\mathcal{B}$ is a square matrix and $\mathcal{C}$ is a column matrix of order twelve. The expressions of the elements of these matrices are given in Appendix 3. The elements of the column matrix $\mathcal{Z}$ are the complex ratios given by $Z_{m} / Z_{o}$ and $Z_{m}^{\prime} / Z_{o}$.

\subsection{Energy ratios}

Now we discuss the distribution of energy of incident wave into distinct reflected and transmitted waves. The rate at which energy is communicated per unit area of surface is given by time averaged energy flux $F$. Since the medium is dissipative and inhomogeneous waves are propagating, so interference of waves would involve the interaction energy among dissimilar waves (Borcherdt 1977; Krebes 1983; Dutta and Ode 1983). The interaction energy among dissimilar waves is the product of stress vector of one wave with velocity vector of another wave. The net energy flux is the sum of orthodox and interference energy fluxes. Therefore, we can write

$$
\left(F, F^{\prime}\right)=\sum_{m} \sum_{n}\left(F_{m n}, F_{m n}^{\prime}\right)
$$

For $F, 0 \leq m, n \leq 6$ and for $F^{\prime}, 1 \leq m, n \leq 6$. The orthodox and interference energy fluxes are given by $F_{m m}$ and $F_{m n}$, respectively. The energy flux due to incident wave is measured by $F_{o}=$ $F_{00}+\sum_{m=1}^{6}\left(F_{0 m}+F_{m 0}\right)$. Expression to calculate orthodox and interference energy fluxes may be written as:

$$
\begin{aligned}
F_{m n}= & -\frac{\omega}{2 \pi} \int_{0}^{\frac{2 \pi}{\omega}}\left(\Re\left(\sigma_{z r, m}^{s_{1}}\right) \Re\left(v_{r, n}^{s_{1}}\right)\right. \\
& +\Re\left(\sigma_{z r, m}^{s_{2}}\right) \Re\left(v_{r, n}^{s_{2}}\right)+\Re\left(\Gamma_{m}^{1}\right) \Re\left(w_{z, n}^{1}\right) \\
& \left.+\Re\left(\Gamma_{m}^{2}\right) \Re\left(w_{z, n}^{2}\right)\right) d t, \quad(r=x, z) .
\end{aligned}
$$

The energy ratios of reflected and transmitted waves to that of incident wave are given by

$$
E_{m n}=\frac{F_{m n}}{F_{00}}, \quad E_{m n}^{\prime}=\frac{F_{m n}^{\prime}}{F_{00}} .
$$

\section{Numerical example}

To study in detail the dependence of reflection coefficients on material parameters of the system, we consider composite porous media composed of sandstone and ice. The pores of the media are assumed to be saturated by oil and water. Ice in the composite rock frame is formed by freezing of water and cementation with sandstone at decreasing temperature. Permafrost is an example of such system and is usually found in cold regions. The interface between two media is defined by increase in rigidity of solid matrix. The parametric values for such a system are given in table 1 (Leclaire et al. 1994; Lo et al. 2005; Denneman et al. 2002).

Numerically we consider that inhomogeneous $P 1$ wave after travelling through the upper medium $\Omega$ strikes at interface of two composite porous media. Interfacial pores are considered to be partially sealed. This situation may appear due to non-connectivity of pores. Therefore, for numerical calculations we stipulate $\varsigma=0.5$. Surface flow impedance for oil and water is assumed to be $T_{1}=1 \mathrm{M} \mathrm{Pas} / \mathrm{m}$ and $T_{2}=10 \mathrm{KPas} / \mathrm{m}$, respectively. Using these parametric values, we solve the system of equations given by equation (40) and obtain complex ratios given by $Z_{m} / Z_{o}$ and $Z_{m}^{\prime} / Z_{o}$. Modulus of these values yield amplitude ratio of corresponding wave.

Variation in amplitude ratios of reflected and transmitted waves with angle of incidence at stipulated level of water saturation and sandstone fraction $\left(S_{2}=S_{s_{2}}=0.5\right)$ in composite matrix are depicted in figure 3 . The wave excitation frequency is also fixed at $1 \mathrm{KHz}$. The angle of attenuation $\left(\gamma_{o}\right)$ of an incident wave is considered to be $40^{\circ}$. This figure implies that amplitude ratios vary continuously with the angle of incidence.

Figure 3(a) depicts that reflection coefficient of $P 1$ wave decreases gradually with the increase in angle of incidence. It attains its minimum value at $\theta_{o}=50^{\circ}$. Further rise in incidence angle increases the value of its amplitude ratio. Near the grazing incidence of an incident wave, reflection coefficient of this wave attains its maximum value. The variation in amplitude ratios of $P 2$ and $P 3$ waves is 
Table 1. Values of parameters.

\begin{tabular}{lll}
\hline Parameters & \multicolumn{1}{c}{$\Omega^{\prime}$} & $\Omega$ \\
\hline Bulk modulus of ice $\left(K_{s_{1}}\right)$ & $8.5 \mathrm{Gpa}$ & $8.5 \mathrm{Gpa}$ \\
Bulk modulus of sandstone $\left(K_{s_{2}}\right)$ & $40 \mathrm{Gpa}$ & $35 \mathrm{Gpa}$ \\
Bulk modulus of oil $\left(K_{1}\right)$ & $0.57 \mathrm{Gpa}$ & $0.57 \mathrm{Gpa}$ \\
Bulk modulus of water $\left(K_{2}\right)$ & $2.25 \mathrm{Gpa}$ & $2.25 \mathrm{Gpa}$ \\
Density of ice $\left(\rho_{s_{1}}\right)$ & $920 \mathrm{~kg} / \mathrm{m}^{3}$ & $920 \mathrm{~kg} / \mathrm{m}^{3}$ \\
Density of sandstone $\left(\rho_{s_{2}}\right)$ & $2900 \mathrm{~kg} / \mathrm{m}^{3}$ & $2650 \mathrm{~kg} / \mathrm{m}^{3}$ \\
Density of oil $\left(\rho_{1}\right)$ & $762 \mathrm{~kg} / \mathrm{m}^{3}$ & $762 \mathrm{~kg} / \mathrm{m}^{3}$ \\
Density of water $\left(\rho_{2}\right)$ & $1000 \mathrm{~kg} / \mathrm{m}^{3}$ & $1000 \mathrm{~kg} / \mathrm{m}^{3}$ \\
Drained bulk modulus of ice $\left(K_{s_{1}}\right)$ & $25 \mathrm{Mpa}$ & $25 \mathrm{Mpa}$ \\
Drained bulk modulus of sandstone $\left(K_{s_{2}}^{d}\right)$ & $83.3 \mathrm{Mpa}$ & $83.3 \mathrm{Mpa}$ \\
Shear modulus of ice $\left(G_{s_{1}}\right)$ & $3.6 \mathrm{Gpa}$ & $3.6 \mathrm{Gpa}$ \\
Shear modulus of sandstone $\left(G_{s_{2}}\right)$ & $3.6 \mathrm{Gpa}$ & $3.85 \mathrm{Mpa}$ \\
Permeability of ice $\left(\mathbb{K}_{s_{1}}\right)$ & $0.107 \mu \mathrm{m}^{2}$ & $0.107 \mu \mathrm{m}^{2}$ \\
Permeability of sandstone $\left(\mathbb{K}_{s_{2}}\right)$ & $0.5 \mathrm{Pico} \mathrm{\textrm {m } ^ { 2 }}$ & $8 \mathrm{Pico} \mathrm{m}$ \\
Viscosity of oil $\left(\eta_{1}\right)$ & $1440 \mu \mathrm{ms}^{2} / \mathrm{m}^{2}$ & $1440 \mu \mathrm{Ns} / \mathrm{m}^{2}$ \\
Viscosity of water $\left(\eta_{2}\right)$ & $0.001 \mathrm{Ns} / \mathrm{m}^{2}$ & $0.001 \mathrm{Ns} / \mathrm{m}^{2}$ \\
Porosity & 0.32 & 0.30 \\
Fitting parameters & & 1.56 \\
$\mathfrak{h}$ & 1.56 & 0.45 \\
$\eta$ & 0.45 &
\end{tabular}
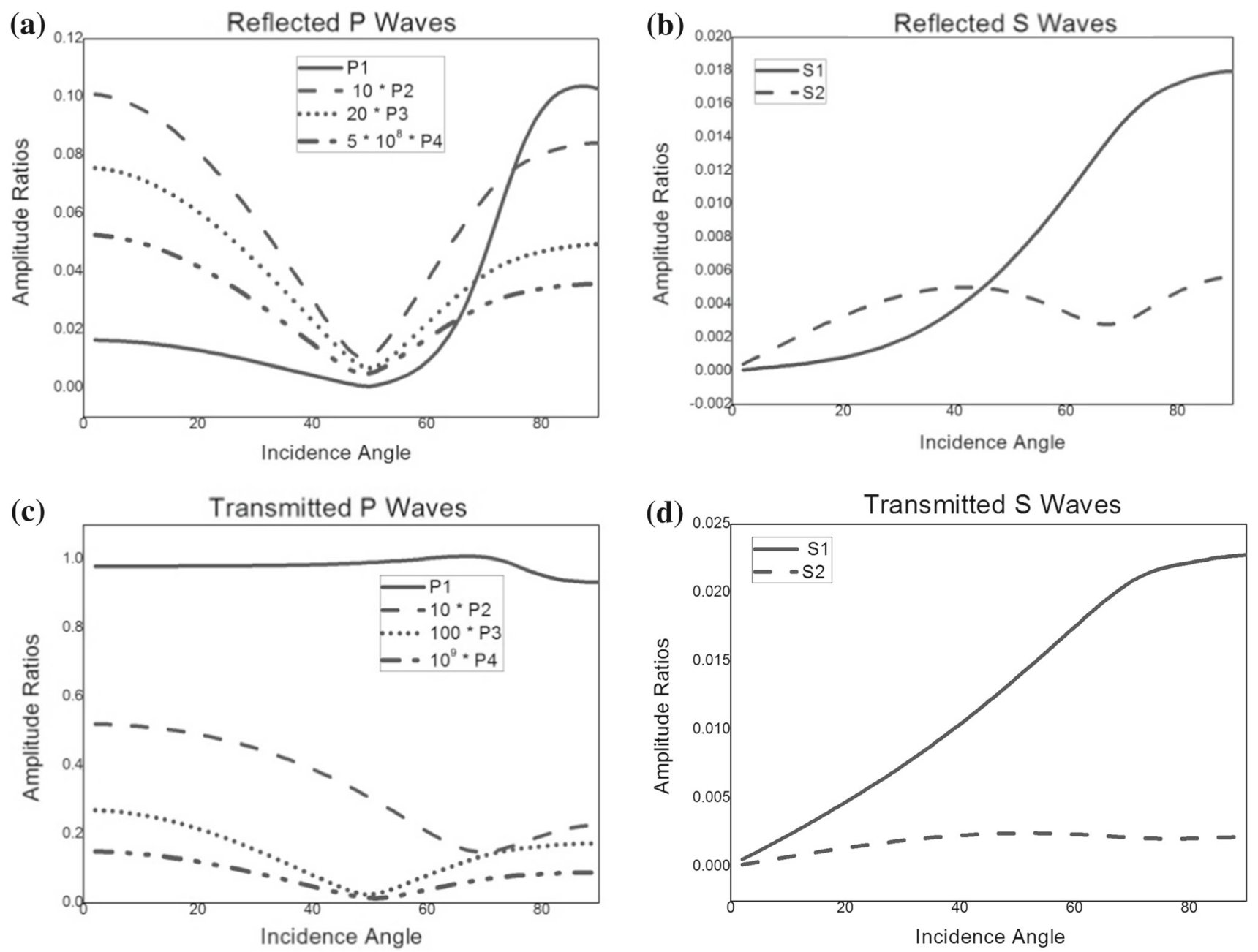

Figure 3. The variation of amplitude ratios of reflected and transmitted waves with incidence angle at $\left(S_{s_{2}}=S_{2}=0.5\right)$. 

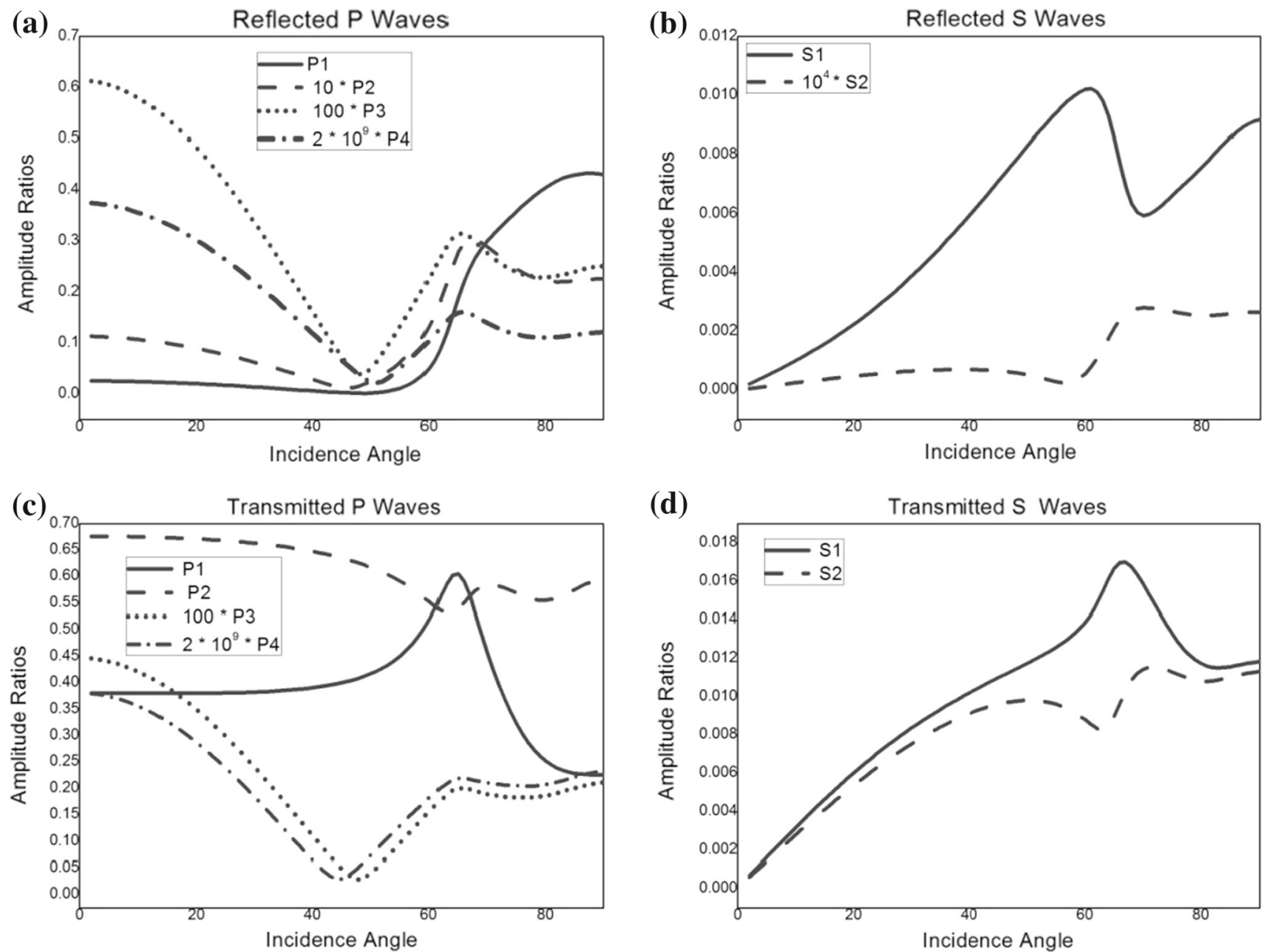

Figure 4. The variation of amplitude ratios of reflected and transmitted waves with incidence angle at $\left(S_{s_{2}}=0.3, S_{2}=0.5\right)$.

similar to $P 1$ wave. A gradual decrease in the values of reflection coefficients of these waves is observed for $0^{\circ} \leq \theta_{0} \leq 50^{\circ}$. Further increase in angle of incidence also increases the values of reflection coefficients of these waves. It is depicted that near normal incidence amplitude ratios of these waves attain maximum value. Capillary pressure wave $(P 4)$, which appears due to presence of second fluid in the medium has very small value of reflection coefficient throughout the entire range of $\theta_{0}$.

The behaviour of amplitude ratios of reflected $S V$ waves is depicted in figure 3(b). It is observed that the increase in incidence angle increases reflection coefficients of both waves. But amplitude ratio of $S 2$ wave is very small as compared to $S 1$ wave. The coefficient of reflected $S 1$ wave attain its maximum value at $\theta_{o} \approx 90^{\circ}$.

When incident $P 1$ wave strikes at interface, the behaviour of transmission coefficients of $P$ waves in $\Omega^{\prime}$ are depicted in figure 3(c). The transmission coefficient of $P 1$ wave is maximum throughout the entire region of angle of incidence. The transmission coefficient of $P 2$ wave decreases continuously and attains its minimum value at $\theta_{o}=70^{\circ}$. The reflection and transmission coefficients of $P 3$ and $P 4$ waves behave in a similar manner. The values of transmission coefficient of capillary pressure wave are very small throughout the entire range of $0 \leq \theta_{o} \leq 90^{\circ}$. Figures 4 and 5 depict the variation in amplitude ratios of different waves at different values of the fraction of sandstone. It is noticeable that with the softening of material reflection amplitudes decreases. Therefore the decrease in ice and increase in the content of sandstone favour the transmission of $P 1$ wave and the incident inhomogeneous $P 1$ wave is mostly converted into transmitted $P 1$ wave. It is also observable that appearance of $S 2$ wave is significant only in case of equal fractions of both the solids in a matrix. 
(a)

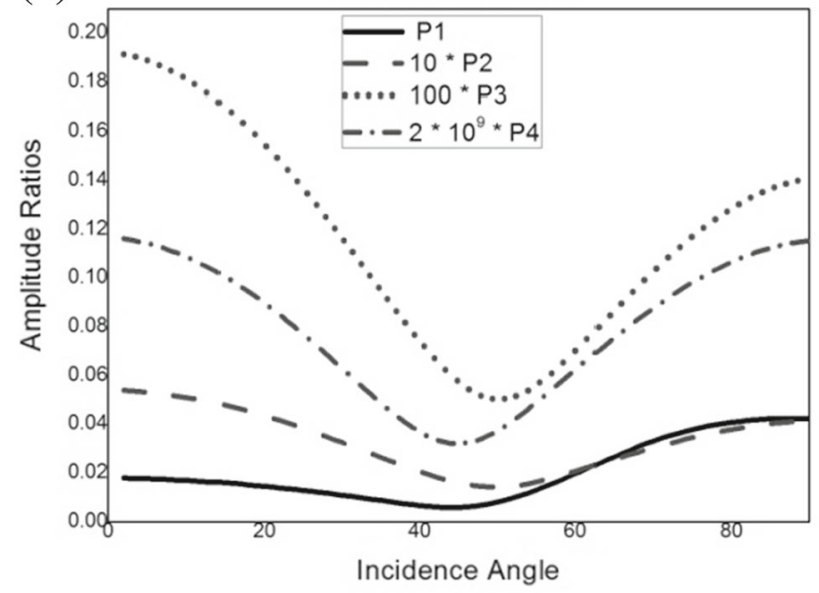

(c)

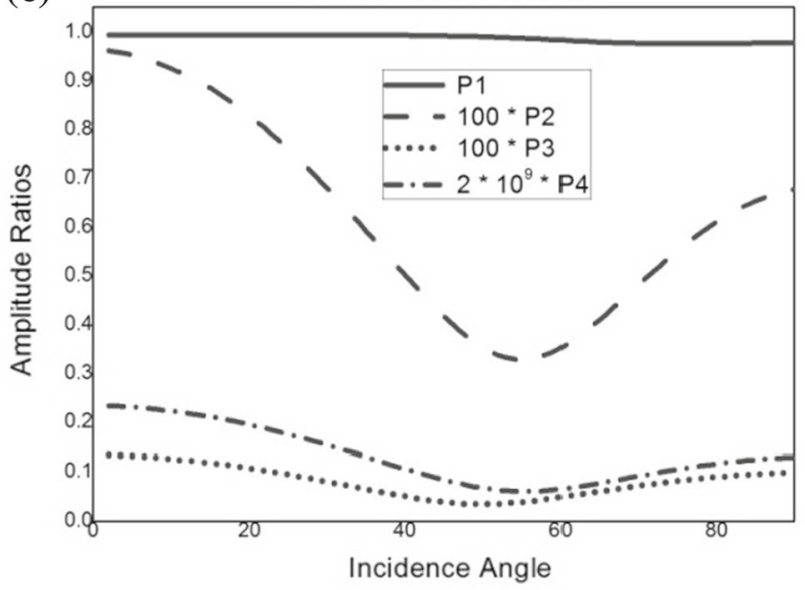

(b) Reflected S Waves

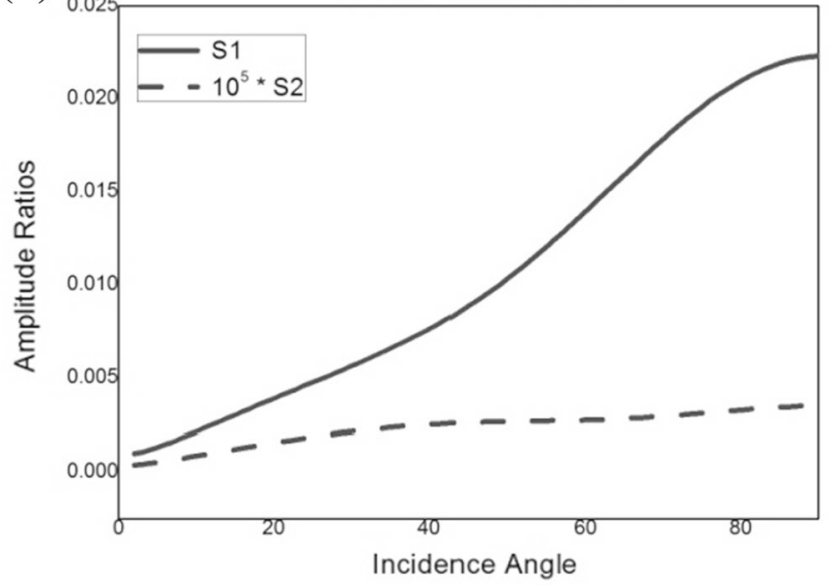

(d)

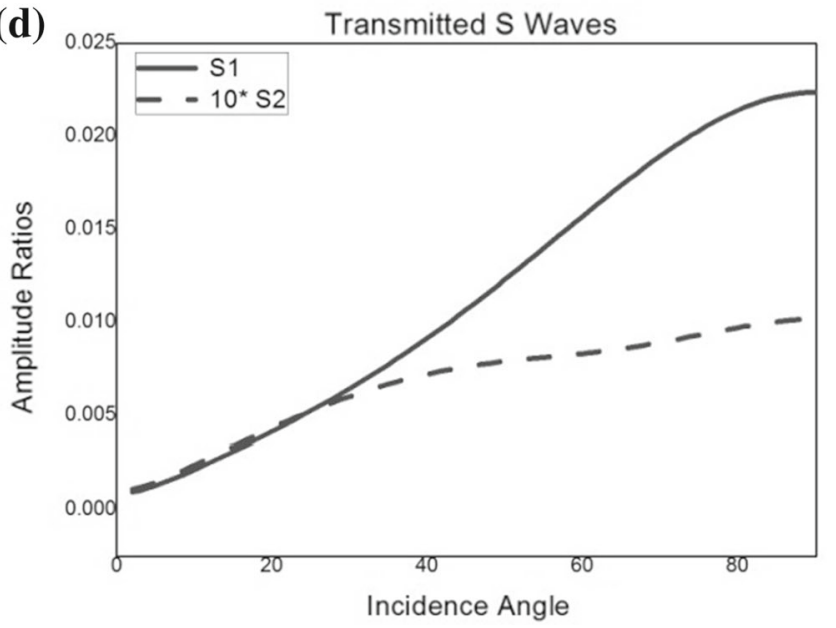

Figure 5. The variation of amplitude ratios of reflected and transmitted waves with incidence angle at $\left(S_{s_{2}}=0.7, S_{2}=0.5\right)$.

The effect of pore connectivity on reflection and transmission coefficients is given in figure $8(\mathrm{a}-$ d) at stipulated $S_{s_{2}}=S_{2}=0.5$. Porosities of both of the media in this case are assumed to be same $\left(f=f^{\prime}=0.32\right)$ and connecting parameter $\varsigma$ assumes the values in $[0,1]$ as $\ell$ vary from 0 to 1 . It is depicted from figure $6(\mathrm{a}-\mathrm{d})$ that the reflection coefficients of $P$ waves increase considerably with little change in value of $\varsigma$ from 0 to 0.1. This implies that sudden change of connecting parameter from non-connectivity of pores to small connectivity increases the reflection coefficients of dilatational waves. But further continuous increase in connectivity does not make significant effect on the reflection coefficients of these waves. This is due to the sudden change in pressure drop at the interface and is in accordance with the observations made by Sharma and Saini (1992). It is also suggested by the boundary conditions (32), that the connection of small portion of pores results into large reduction in pressure at the interface. Mathematically, $\varsigma \rightarrow 0$ implies $\Gamma^{\mathfrak{f}} \rightarrow \infty$, therefore even a $10 \%$ opening of pores reduces the interfacial pressure to greater extent. It may be concluded that reflection and transmission coefficients depend upon opening of surface pores and not on the extent of openness. The variation in transmission coefficients of $P$ waves with connecting parameter decreases with the pressure drop and show exactly reverse trends as shown by reflection coefficients of these waves. Transmission coefficients of $S V$ waves show the similar trends as shown by their reflection coefficients at all angle of incidence and at all values of connecting parameter.

Variation in energy ratios with incidence angle $\left(\theta_{0}\right)$ at stipulated sandstone fraction and water saturation $S_{s_{2}}=S_{2}=0.5$, are given in figure $7(\mathrm{a}-\mathrm{d})$. At each angle of incidence the incident energy is found to be sum of energy ratios of reflected, refracted waves and interacting energies. 
(a)

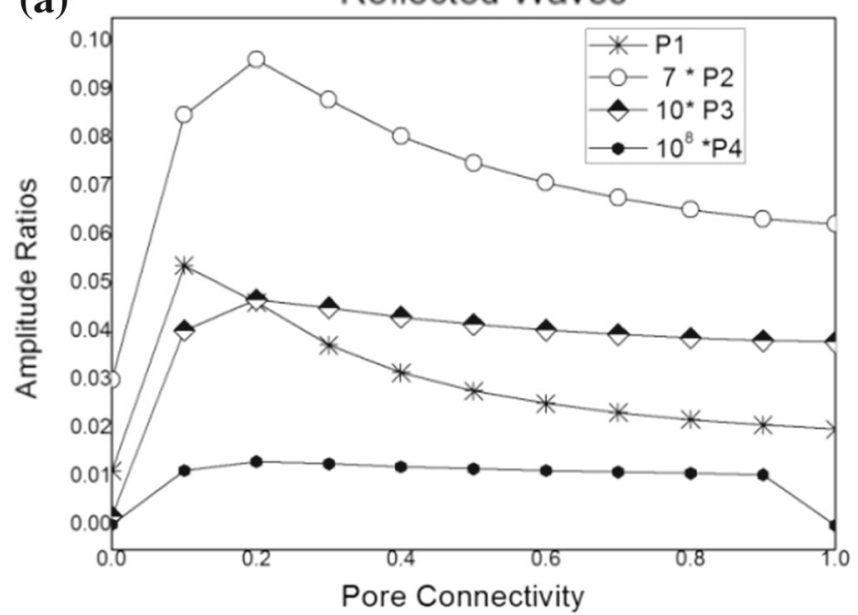

(c)

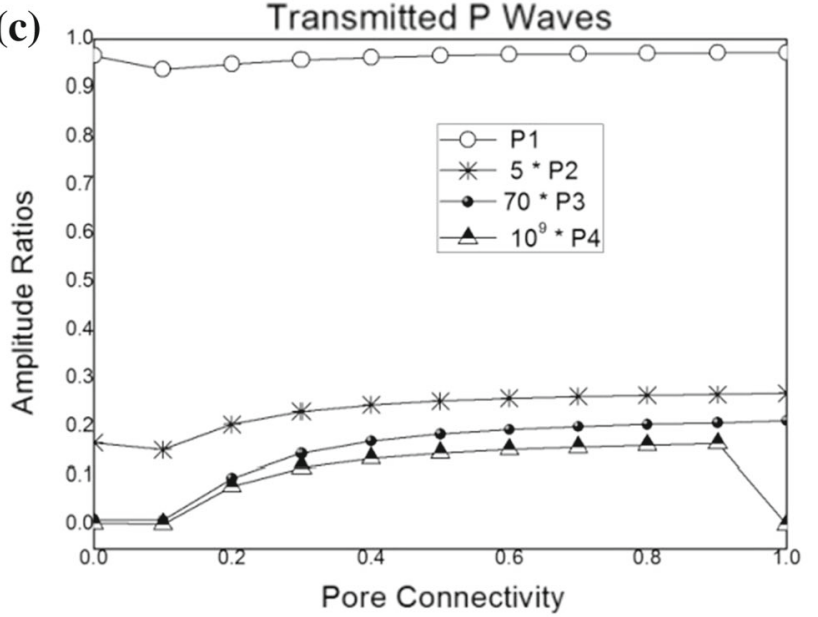

(b)

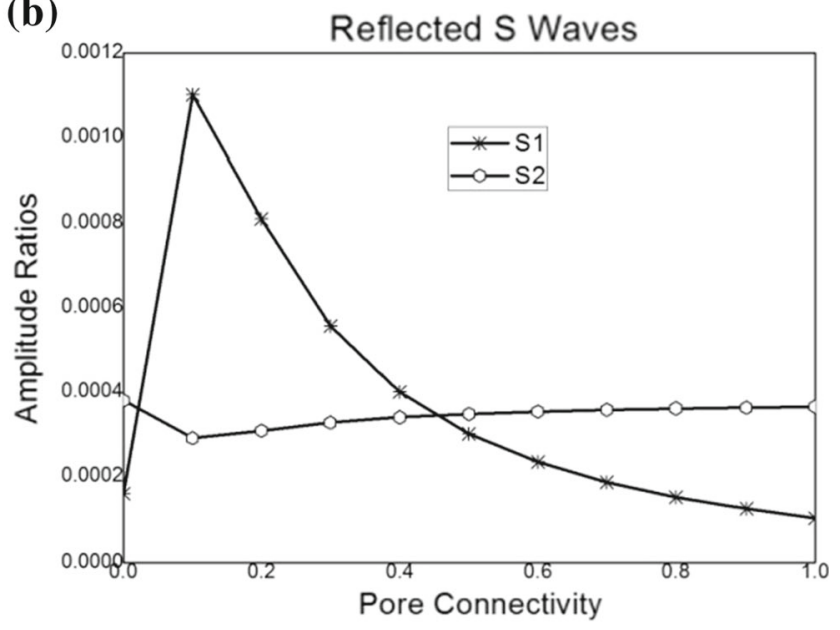

(d)

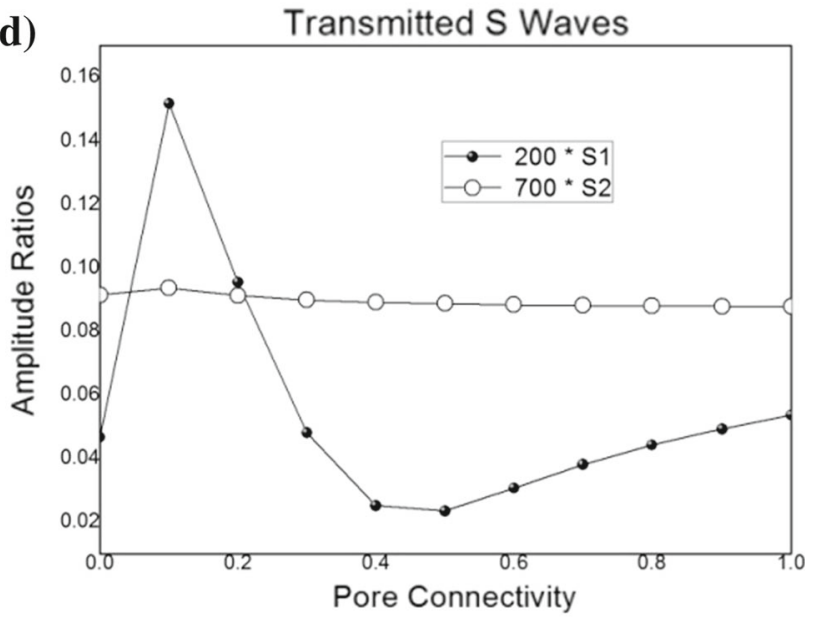

Figure 6. The variation of amplitude ratios of reflected and transmitted waves with pore connectivity.

Since energy ratios are proportional to square of amplitude ratios, trends shown by energy ratios are similar to amplitude ratios. It is noticed that maximum energy of an incident wave is carried by the transmitted $P 1$ wave. Minimum energy is transferred to capillary pressure wave at interface.

In $\Omega$ total interaction, energy $\left(E_{I 1}\right)$ is the sum of energy ratios appearing due to interference among incident and reflected waves, and due to interference of reflected waves themselves. The interaction energy $E_{I 2}$ corresponds to the sum of energy ratios due to interference among transmitted waves in $\Omega^{\prime}$. Sum of interaction energies $\left(E_{I 1}+E_{I 2}\right)$ contributes towards the conservation of energy at interface. Variations in $E_{I 1}$ and $E_{I 2}$ with angle of incidence are respectively depicted by figure $7(\mathrm{e})$. It is noticeable that interference of waves in both the media is maximum in $40^{\circ} \leq \theta_{o} \leq 60^{\circ}$.

The equations of our model are reduced to single solid system by assuming appropriate parameters equal to zero. The variation in amplitude ratios of four waves so obtained were compared with ratios obtained by introducing following Zoeppritz equations.

$$
\begin{gathered}
{\left[\begin{array}{cccc}
-\sin \theta_{o} & -\cos \theta_{5} & \sin \theta_{1}^{\prime} & \cos \theta_{5}^{\prime} \\
\cos \theta_{o} & -\sin \theta_{5} & \cos \theta_{1}^{\prime} & -\sin \theta_{5}^{\prime} \\
\sin 2 \theta_{o} & \varrho_{1} \cos 2 \theta_{5} & \varrho_{2} \cos 2 \theta_{5} & \varrho_{3} \cos 2 \theta_{5}^{\prime} \\
-\cos 2 \theta_{5} & \frac{1}{\varrho_{1}} \sin 2 \theta_{5} & \varrho_{4} \cos 2 \theta_{5}^{\prime} & \varrho_{5} \sin 2 \theta_{5}^{\prime}
\end{array}\right]} \\
\times\left[\begin{array}{l}
R_{P} \\
R_{S} \\
T_{P} \\
T_{S}
\end{array}\right]=\left[\begin{array}{c}
\sin \theta_{o} \\
\cos \theta_{o} \\
\sin 2 \theta_{o} \\
\cos 2 \theta_{5}
\end{array}\right],
\end{gathered}
$$

where $\varrho_{1}=\frac{v_{1}}{v_{5}}, \varrho_{2}=\frac{\rho_{s_{2}}^{\prime} v_{5}^{\prime 2} v_{1}}{\rho_{s_{2}} v_{5}^{2} v_{1}^{\prime}}, \varrho_{3}=\frac{\rho_{s_{2}}^{\prime} v_{5}^{\prime} v_{1}}{\rho_{s_{2}} v_{5}^{2}}$, $\varrho_{4}=\frac{\rho_{s_{2}}^{\prime} v_{1}^{\prime}}{\rho_{s_{2}} v_{1}}, \varrho_{5}=\frac{\rho_{s_{2}}^{\prime} v_{5}^{\prime}}{\rho_{s_{2}} v_{1}}$. The symbols $R_{P}, T_{P}$, 
(a)

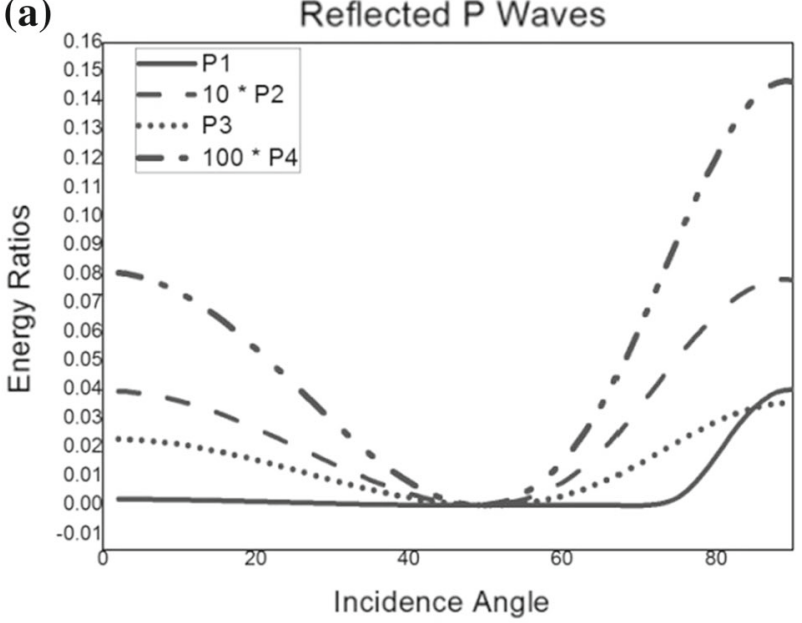

(c)
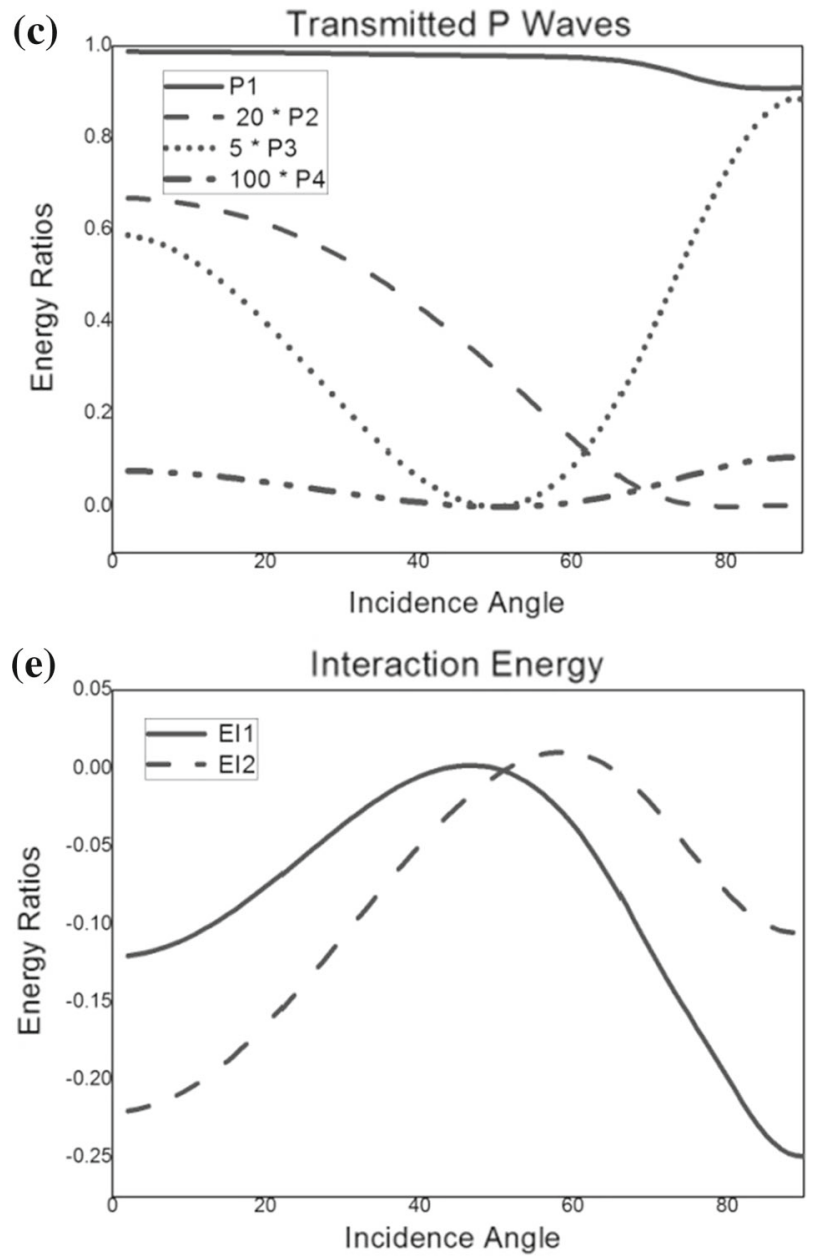

(b)

Reflected S Waves

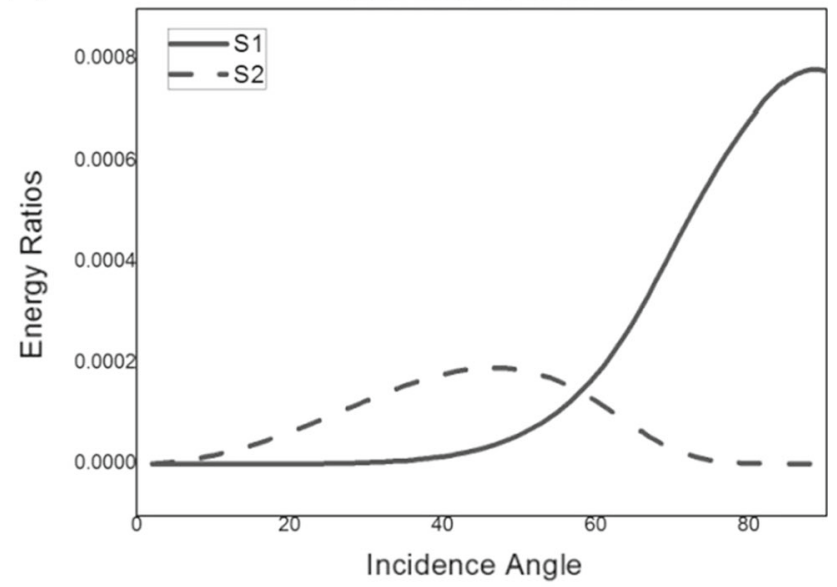

(d)

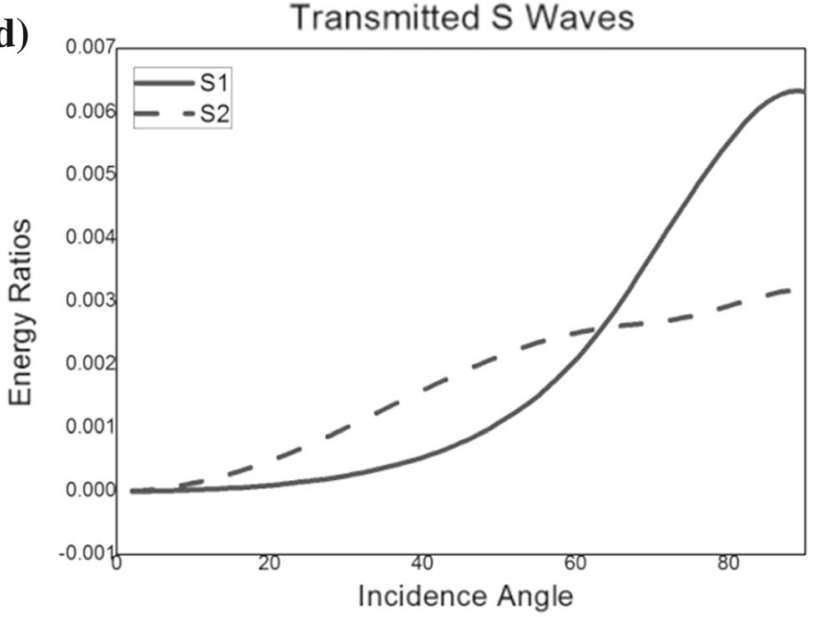

Figure 7. The variation of energy ratios of reflected and transmitted waves with incidence angle.

$R_{S}$ and $T_{S}$ represent reflected and transmitted coefficients of $P$ and $S$ waves, respectively. The graphical comparison of reflected and refracted waves at stipulated frequency $1 \mathrm{KHz}$ is given in figure 8. For numerical calculations, we consider the two media to be composed of distinct sandstones whose parameters are given in table 1 . Figure $8(\mathrm{a}-\mathrm{b})$ depicts the variation of reflected and refracted $P$ waves at interface. It is observed that the variation of reflected and refracted $P$ waves with angle of incidence is similar in both models. It is observed that the smoothness of amplitude ratio curve of all the reflected and transmitted waves is disturbed at $\theta_{o} \approx 67^{\circ}$, which is observed to be the critical angle of transmitted $P$ wave. 

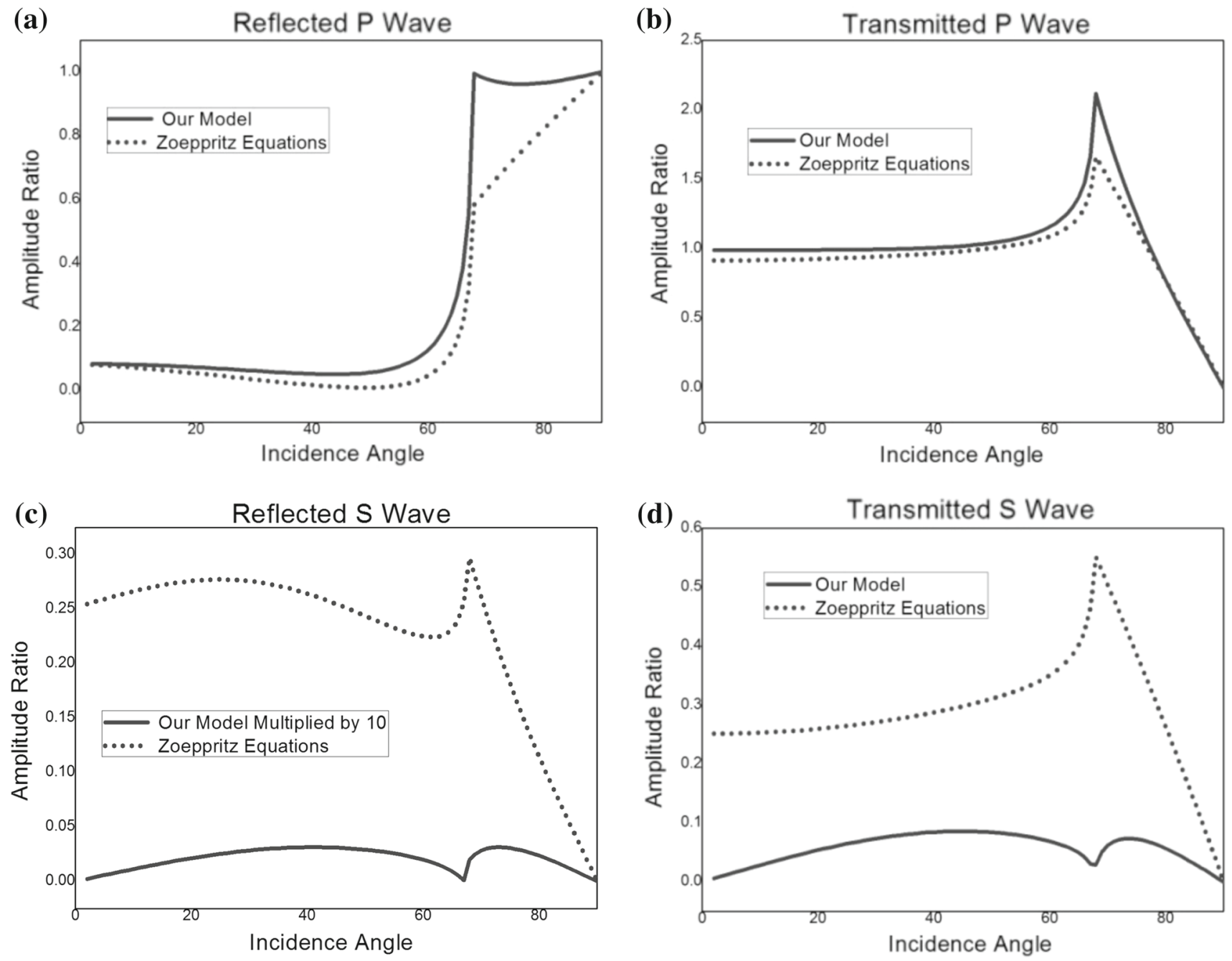

Figure 8. Comparison of amplitude ratios with the ratios obtained using Zoeppritz equations.

\section{Conclusion}

A study of the phenomenon of reflection and transmission is conducted when a general inhomogeneous wave impinges at the interface between two distinct porous media. Both the media are assumed to be composed of two solids and saturated by two immiscible fluids. It is observed that incident wave scatters into 12 reflected and transmitted waves. Four dilatational and two transverse waves are reflected back into the medium of incident wave, while four dilatational and two transverse waves are transmitted. The expression of Umov-Poynting vector is derived to find energy fluxes along the normal to the interface and to frame required boundary conditions. The effect of variability of connection among pores at the interface is analyzed on the reflection amplitudes and energy ratios. Partition of incident energy among reflected and transmitted waves is calculated along with the consideration of interaction energy due to interference of distinct waves. The dependance of reflection and energy ratios on different parameters of the media are studied for the composite material composed of sandstone and ice and saturated with oil and water. Some interesting and important observations based on numerical example are listed below

1. Reflection amplitude of $P 1$ wave has maximum value at nearly grazing incidence of wave whereas all other reflected $P$ waves dominate near normal incidence of wave. Reflection amplitude of this wave is more apparent in a composite matrix dominantly composed of ice.

2. Reflection amplitudes of $S V$ waves also appear more comfortably near $\theta_{o}=90^{\circ}$. The reflection coefficient of $S 2$ wave is very small as compared to $S 1$ wave and appears significantly only in case of a composite matrix composed of equal fraction of sandstone and ice and equally saturated by oil and water. 
3. Transmission coefficient of dilatational $P 1$ wave dominates with the increase in the fraction of sandstone. This implies that with the softening of a rock the most of the energy of incident $P 1$ wave is transformed into the energy of a transmitted $P 1$ wave.

4. The capillary pressure wave $P 4$ appears due to the presence of two fluids in the medium. Values of amplitude ratio of this wave are very small throughout the entire range of $\theta_{o}$.

5. Transmission coefficients of transverse waves dominate over their reflection coefficients almost throughout the entire range of study.

6. Sudden change in the value of connecting parameter $\varsigma$ from 0 to 0.1 , that is, from nonconnectivity of pores to slight connectivity greatly influences the scattering of wave at the interface.

\section{Acknowledgement}

One of the authors (AA) is tankful to the Council of Scientific and Industrial Research (CSIR), New Delhi for providing financial support under Scheme No. 25(0243)/15/EMR-II to complete this work.

\section{Appendix 1}

(a) The elements of matrices appearing in equation

(2) are given below.

$$
\begin{aligned}
& {[\sigma]=\left[\begin{array}{llllll}
\sigma_{x x}^{s_{1}} & \sigma_{y y}^{s_{1}} & \sigma_{z z}^{s_{1}} & \sigma_{x y}^{s_{1}} & \sigma_{x z}^{s_{1}} & \sigma_{y z}^{s_{1}}
\end{array}\right.}
\end{aligned}
$$

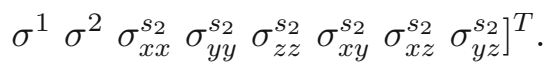

$$
\begin{aligned}
& {[\epsilon]=\left[\epsilon_{x x}^{s_{1}} \epsilon_{y y}^{s_{1}} \epsilon_{z z}^{s_{1}} \epsilon_{x y}^{s_{1}} \epsilon_{x z}^{s_{1}}\right.}
\end{aligned}
$$

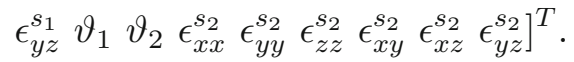

Non-zero elements of symmetric matrix $[\mathcal{L}]$ are

$$
\begin{aligned}
& \mathcal{L}_{1,1}=\mathcal{L}_{2,2}=\mathcal{L}_{3,3}=\bar{a}_{11}, \\
& \mathcal{L}_{1,2}=\mathcal{L}_{1,3}=\mathcal{L}_{2,3}=\breve{a}_{11}, \\
& \mathcal{L}_{1,7}=\mathcal{L}_{2,7}=\mathcal{L}_{3,7}=a_{12}, \\
& \mathcal{L}_{1,8}=\mathcal{L}_{2,8}=\mathcal{L}_{3,8}=a_{13}, \\
& \mathcal{L}_{1,9}=\mathcal{L}_{2,9}=\mathcal{L}_{3,9}=a_{14}, \\
& \mathcal{L}_{1,10}=\mathcal{L}_{2,10}=\mathcal{L}_{3,10}=a_{14}, \\
& \mathcal{L}_{1,11}=\mathcal{L}_{2,11}=\mathcal{L}_{3,11}=a_{14}, \\
& \mathcal{L}_{4,4}=\mathcal{L}_{5,5}=\mathcal{L}_{6,6}=G_{s_{1}}, \\
& \mathcal{L}_{7,7}=a_{22}, \mathcal{L}_{7,8}=a_{23}, \\
& \mathcal{L}_{7,9}=\mathcal{L}_{7,10}=\mathcal{L}_{7,11}=a_{24},
\end{aligned}
$$

$$
\begin{aligned}
& \mathcal{L}_{8,8}=a_{33}, \mathcal{L}_{8,9}=\mathcal{L}_{8,10}=\mathcal{L}_{8,11}=a_{34}, \\
& \mathcal{L}_{9,9}=\mathcal{L}_{10,10}=\mathcal{L}_{11,11}=\bar{a}_{44}, \\
& \mathcal{L}_{9,10}=\mathcal{L}_{9,11}=\mathcal{L}_{10,11}=\breve{a}_{44}, \\
& \mathcal{L}_{12,12}=\mathcal{L}_{13,13}=\mathcal{L}_{14,14}=G_{s_{2}},
\end{aligned}
$$

where

$$
\begin{aligned}
& \vartheta_{\mathfrak{f}}=\nabla \cdot \boldsymbol{u}_{\mathfrak{f}}, \breve{a}_{11}=a_{11}-\frac{2}{3} G_{s_{1}}, \breve{a}_{44}=a_{44}-\frac{2}{3} G_{s_{2}}, \\
& a_{11}=K_{s_{1}} S_{s_{1}}\left(-\delta_{s_{1}}+\theta_{s}\right), \quad a_{12}=-K_{s_{1}} S_{s_{1}} \delta_{1} \text {, } \\
& a_{13}=-K_{s_{1}} S_{s_{1}} \delta_{2}, \quad a_{34}=-K_{s_{2}} S_{s_{2}} \delta_{2} \text {, } \\
& a_{14}=-K_{s_{1}} S_{s_{1}} \delta_{s_{2}}=-K_{s_{2}} S_{s_{2}} \delta_{s_{1}} \text {, } \\
& a_{22}=\frac{-1}{M_{1}}\left[M_{3} \delta_{1}+\frac{K_{1} K_{2} S_{1} f}{1-S_{1}} \frac{d S_{1}}{d p_{c}}+K_{1} f S_{1}\right] \text {, } \\
& a_{23}=\frac{-1}{M_{1}}\left[\frac{\delta_{1} \delta_{2} M_{1}\left(K_{s_{1}} S_{s_{1}}+K_{s_{2}} S_{s_{2}}\right)}{\delta_{s_{1}}+\delta_{s_{2}}}\right. \\
& \left.+K_{1} K_{2} f \frac{d S_{1}}{d p_{c}}\right], \\
& a_{24}=-K_{s_{2}} S_{s_{2}} \delta_{1} \text {, } \\
& a_{33}=\frac{-1}{M_{1}}\left[M_{4} \delta_{2}+\frac{K_{1} K_{2}\left(1-S_{1}\right) f}{S_{1}} \frac{d S_{1}}{d p_{c}}\right. \\
& \left.+K_{2}\left(1-S_{1}\right) f\right] \text {, } \\
& a_{44}=K_{s_{2}} S_{s_{2}}\left(-\delta_{s_{2}}+\theta_{s}\right) \text {, } \\
& \delta_{1}=\frac{M_{3}}{M_{1}\left(K_{s_{1}} S_{s_{1}}+K_{s_{2}} S_{s_{2}}\right)}\left(\delta_{s_{1}}+\delta_{s_{2}}\right), \\
& M_{1}=-\left(1+\frac{K_{2}}{1-S_{1}} \frac{d S_{1}}{d p_{c}}+\frac{K_{1}}{S_{1}} \frac{d S_{1}}{d p_{c}}\right) \text {, } \\
& \delta_{2}=\frac{M_{4}}{M_{1}\left(K_{s_{1}} S_{s_{1}}+K_{s_{2}} S_{s_{2}}\right)}\left(\delta_{s_{1}}+\delta_{s_{2}}\right) \text {, } \\
& M_{2}=\frac{K_{1} K_{2}}{f S_{1}\left(1-S_{1}\right)} \frac{d S_{1}}{d p_{c}}+\frac{K_{1} S_{1}}{f}+\frac{K_{2}\left(1-S_{1}\right)}{f}, \\
& M_{3}=\frac{K_{1} K_{2} S_{1}}{1-S_{1}} \frac{d S_{1}}{d p_{c}}+K_{1} S_{1}+K_{1} K_{2} \frac{d S_{1}}{d p_{c}}, \\
& M_{4}=K_{1} K_{2} \frac{d S_{1}}{d p_{c}}+K_{2}\left(\frac{K_{1}}{S_{1}} \frac{d S_{1}}{d p_{c}}+1\right)\left(1-S_{1}\right) \text {, } \\
& \delta_{s_{f}}=K_{s_{f}}\left(\frac{-1}{K_{s_{f}}}+\frac{\theta_{s_{f}}}{K_{s_{f}}^{\prime}}\right)\left[\mathcal{B}+\frac{M_{2}\left(1-\frac{K_{s_{f}} \theta_{s_{f}}}{K_{s_{f}}^{\prime}}\right)}{M_{1} K_{s_{f}} S_{s_{f}}}\right]^{-1}, \\
& \mathcal{B}=\left(\frac{K_{s_{1}} \theta_{s_{1}}}{K_{s_{1}}^{\prime}}+\frac{K_{s_{2}} \theta_{s_{2}}}{K_{s_{2}}^{\prime}}\right) \frac{1}{1-f}
\end{aligned}
$$

(b) Expressions of coefficients appearing due to inertial and viscous drag

$$
\begin{gathered}
A_{11}=S_{s_{1}}\left(\rho_{1} \theta_{1}-q_{1} f^{2}\right), \\
A_{22}=S_{s_{1}}\left(\rho_{2} \theta_{2}-q_{2} f^{2}\right), \\
B_{11}=S_{s_{2}}\left(\rho_{1} \theta_{1}-q_{1}{ }^{\prime} f^{2}\right),
\end{gathered}
$$




$$
\begin{aligned}
& B_{22}=S_{s_{2}}\left(\rho_{2} \theta_{2}-q_{2}{ }^{\prime} f^{2}\right), \quad A_{12}=-0.1 \sqrt{q_{1} q_{2}{ }^{\prime}}, \\
& R_{11}=\frac{-\theta_{1}^{2} \eta_{1}}{\mathbb{K}_{s} \mathbb{K}_{r 1}}, \quad R_{22}=\frac{-\theta_{2}^{2} \eta_{2}}{\mathbb{K}_{s} \mathbb{K}_{r 2}}, \\
& R_{12}=0, q_{\mathfrak{f}}=\frac{\mathfrak{T}_{s_{1}} \rho_{\mathfrak{f}} S_{\mathfrak{f}}}{f}, q_{\mathfrak{f}}{ }^{\prime}=\frac{\mathfrak{T}_{s_{2}} \rho_{\mathfrak{f}} S_{\mathfrak{f}}}{f}, \\
& \mathbb{K}_{s}=S_{s_{1}} \mathbb{K}_{s_{1}}+S_{s_{2}} \mathbb{K}_{s_{2}}, \\
& \mathbb{K}_{r 1}\left(S_{2}\right)=\left(1-S_{2}\right)^{\eta}\left[1-\left(S_{2}\right)^{\frac{1}{\mathfrak{g}}}\right]^{2 \mathfrak{g}}, \\
& \mathbb{K}_{r 2}\left(S_{2}\right)=\left(S_{2}\right)^{\eta}\left[\left(1-\left(1-\left(S_{2}\right)^{\frac{1}{\mathfrak{g}}}\right)\right)^{\mathfrak{g}}\right]^{2}, \\
& \frac{d p_{c}}{d S_{1}}=\frac{1}{\mathfrak{g h \mathfrak { i }}}\left[\left(1-S_{1}\right)^{\frac{-\mathfrak{h}}{\mathfrak{h}-1}}-1\right]^{\frac{1-\mathfrak{h}}{\mathfrak{h}}}\left(1-S_{1}\right)^{\frac{-(2 \mathfrak{h}-1)}{\mathfrak{h}-1}},
\end{aligned}
$$

where $K_{\alpha}$ represents bulk modulus of each phase. $\mathbb{K}_{s_{1}}$ and $\mathbb{K}_{s_{2}}$ represent permeability of first and second solid, respectively whereas $\eta_{\mathfrak{f}}$ corresponds to viscosity of fluid phases. The symbols $\mathfrak{T}_{s_{1}}, \mathfrak{T}_{s_{2}}$ correspond to the tortuosity of the solids, which depends upon porosity. For numerical purpose their values are obtained from the relation $\mathfrak{T}_{s_{1}}=$ $\mathfrak{T}_{s_{2}}=\frac{1}{2}(1+(1 / f))$. The symbols $S_{\mathfrak{f}}=\theta_{\mathfrak{f}} / f$ and $S_{s_{\mathrm{f}}}=\theta_{s_{\mathrm{f}}} / \theta_{s}, \quad\left(\theta_{s}=\theta_{s_{1}}+\theta_{s_{2}}\right)$ correspond to saturation of fluid phases and solid fraction in composite matrix, respectively. Pore space is considered to be completely filled by fluids. Mathematically, this can be written as $S_{1}+S_{2}=1$. Dry bulk modulus and shear modulus of each solid is depicted by $K_{s_{\mathrm{f}}}^{d}$, $G_{s_{\mathrm{f}}}$. The term $p_{c}$ signifies the pressure difference between fluid phases. For numerical example we consider $\mathfrak{i}=25 \times 10^{-5}$. The symbols $\mathfrak{g}=1-(1 / \mathfrak{h})$, $\mathfrak{h}$ and $\mathfrak{i}$ correspond to fitting parameters involved in understanding the soil water content in Van ganuchten model (1980). The values of these independent parameters may be obtained by finding the best fit of soil water retention curve to experimental data. In most of cases the method of least squares is used to find the value of these parameters. The parameter $\eta$ is also a fitting parameter, which is employed to find the relative permeability of wetting and non-wetting fluids as a function of water saturation.

\section{Appendix 2}

The expressions of coupling coefficients

$$
\begin{aligned}
& \kappa_{1}=\operatorname{Det}\left[\begin{array}{lll}
X_{4} & X_{2} & X_{3}
\end{array}\right], \kappa_{2}=\operatorname{Det}\left[\begin{array}{lll}
X_{1} & X_{4} & X_{3}
\end{array}\right], \\
& \kappa_{3}=\operatorname{Det}\left[\begin{array}{lll}
X_{1} & X_{4} & X_{3}
\end{array}\right], \kappa_{4}=\operatorname{Det}\left[\begin{array}{lll}
X_{1} & X_{2} & X_{3}
\end{array}\right], \\
& \epsilon_{1}=\operatorname{Det}\left[\begin{array}{lll}
X_{8} & X_{6} & X_{7}
\end{array}\right], \epsilon_{2}=\operatorname{Det}\left[\begin{array}{lll}
X_{5} & X_{8} & X_{7}
\end{array}\right], \\
& \epsilon_{3}=\operatorname{Det}\left[\begin{array}{llll}
X_{5} & X_{6} & X_{8}
\end{array}\right], \quad \epsilon_{4}=\operatorname{Det}\left[\begin{array}{llll}
X_{5} & X_{6} & X_{7}
\end{array}\right],
\end{aligned}
$$

where Det is employed to symbolize the determinant of a matrix. Also,

$$
\begin{aligned}
& X_{1}=\left[\begin{array}{lll}
a_{22} Y+\eta_{11} & a_{23} Y+\eta_{12} & a_{24} Y+\eta_{01}
\end{array}\right]^{T}, \\
& X_{2}=\left[\begin{array}{lll}
a_{23} Y+\eta_{12} & a_{33} Y+\eta_{23} & a_{34} Y+\eta_{02}
\end{array}\right]^{T}, \\
& X_{3}=\left[\begin{array}{lll}
a_{24} Y+\eta_{01} & a_{34} Y+\eta_{02} & \bar{a}_{44} Y+\eta_{44}
\end{array}\right]^{T}, \\
& X_{4}=-\left[\begin{array}{lll}
a_{12} Y+\eta_{01} & a_{13} Y+\eta_{02} & a_{14} Y+\eta_{03}
\end{array}\right]^{T}, \\
& X_{5}=\left[\begin{array}{lll}
\eta_{11} & \eta_{12} & \eta_{01}
\end{array}\right]^{T}, X_{6}=\left[\begin{array}{lll}
\eta_{12} & \eta_{23} & \eta_{02}
\end{array}\right]^{T}, \\
& X_{7}=\left[\begin{array}{lll}
\eta_{01} & \eta_{02} & G_{s_{2}} Y+\eta_{44}
\end{array}\right]^{T}, \\
& X_{8}=-\left[\begin{array}{lll}
\eta_{01} & \eta_{02} & \eta_{03}
\end{array}\right]^{T}, \\
& \lambda_{s_{1}}^{\beta}=1, \quad \lambda_{1}^{\beta}=\frac{\kappa_{1}}{\kappa_{4}}, \quad \lambda_{2}^{\beta}=\frac{\kappa_{2}}{\kappa_{4}}, \quad \lambda_{s_{2}}^{\beta}=\frac{\kappa_{3}}{\kappa_{4}}, Y=\frac{-1}{v_{\beta}^{2}}, \\
& \lambda_{s_{1}}^{l}=1, \quad \lambda_{1}^{l}=\frac{\epsilon_{1}}{\epsilon_{4}}, \quad \lambda_{2}^{l}=\frac{\epsilon_{2}}{\epsilon_{4}}, \quad \lambda_{s_{2}}^{l}=\frac{\epsilon_{3}}{\epsilon_{4}}, \quad Y=\frac{-1}{v_{l}^{2}} .
\end{aligned}
$$

\section{Appendix 3}

The explicit expressions of elements of matrix given in equation (40) are

$$
\begin{aligned}
& b_{1, \beta}=-\left(J_{\beta}+\left(2 G_{s_{1}}+J_{\beta}\right) d_{\beta}^{2}\right), \\
& b_{1, l}=2 G_{s_{1}} d_{l}, \quad b_{1, \beta}^{\prime}=J_{\beta}^{\prime}+\left(2 G_{s_{1}}^{\prime}+J_{\beta}^{\prime}\right) d_{\beta}^{\prime 2} \text {, } \\
& b_{1, l}^{\prime}=2 G_{s_{1}}^{\prime} d_{l}^{\prime} \text {, } \\
& b_{2, \beta}=-\left(M_{\beta}+\left(2 G_{s_{2}} \lambda_{s_{2}}^{\beta}+M_{\beta}\right) d_{\beta}^{2}\right), \\
& b_{2, l}=2 \lambda_{s_{2}}^{l} G_{s_{2}} d_{l} \text {, } \\
& b_{2, \beta}^{\prime}=M_{\beta}^{\prime}+\left(2 G_{s_{2}}^{\prime} \lambda_{s_{2}}^{\beta^{\prime}}+M_{\beta}^{\prime}\right) d_{\beta}^{\prime 2} \text {, } \\
& b_{2, l}^{\prime}=2 \lambda_{s_{2}}^{l^{\prime}} G_{s_{2}}^{\prime} d_{l}^{\prime}, \quad b_{3, \beta}=2 G_{s_{1}} d_{\beta} \text {, } \\
& b_{3, l}=G_{s_{1}}\left(-1+d_{l}^{2}\right), \quad b_{3, \beta}^{\prime}=2 G_{s_{1}}^{\prime} d_{\beta}^{\prime} \text {, } \\
& b_{3, l}^{\prime}=-G_{s_{1}}^{\prime}\left(-1+d_{l}^{\prime 2}\right) \text {, } \\
& b_{4, \beta}=2 G_{s_{2}} \lambda_{s_{2}}^{\beta} d_{\beta}, \quad b_{4, l}=G_{s_{2}}\left(-1+d_{l}^{2}\right) \lambda_{s_{2}}^{l}, \\
& b_{4, \beta}^{\prime}=2 G_{s_{2}}^{\prime} \lambda_{s_{2}}^{\beta^{\prime}} d_{\beta}^{\prime} \text {, } \\
& b_{4, l}^{\prime}=-G_{s_{2}}^{\prime}\left(-1+d_{l}^{\prime 2}\right) \lambda_{s_{2}}^{l^{\prime}}, \quad b_{5, \beta}=1, \\
& b_{5, l}=d_{l}, \quad b_{5, \beta}^{\prime}=-1, \quad b_{5, l}^{\prime}=d_{l}^{\prime}, \\
& b_{6, \beta}=\lambda_{s_{2}}^{\beta}, \quad b_{6, l}=\lambda_{s_{2}}^{l} d_{l}, \quad b_{6, \beta}^{\prime}=-\lambda_{s_{2}}^{\beta^{\prime}}, \\
& b_{6, l}^{\prime}=\lambda_{s_{2}}^{l}{ }^{\prime} d_{l}^{\prime}, \quad b_{7, \beta}=-d_{\beta}, \\
& b_{7, l}=1, \quad b_{7, \beta}^{\prime}=-d_{\beta}^{\prime}, \quad b_{7, l}^{\prime}=-1, \\
& b_{8, \beta}=-\lambda_{s_{2}}^{\beta} d_{\beta}, \quad b_{8, l}=\lambda_{s_{2}}^{l}, \\
& b_{8, \beta}^{\prime}=-\lambda_{s_{2}}^{\beta^{\prime}} d_{\beta}^{\prime}, \quad b_{8, l}^{\prime}=-\lambda_{s_{2}}^{l^{\prime}}, \\
& b_{9, \beta}=-\varsigma\left(\delta_{\beta}-\xi_{\beta} \theta_{1}+d_{\beta}^{2}\left\{\delta_{\beta}-\left(H_{\beta}+\xi_{\beta}\right) \theta_{1}\right\}\right) \text {, } \\
& b_{9, l}=-\varsigma d_{l} S_{l} \theta_{1} \text {, } \\
& b_{9, \beta}^{\prime}=(1-\varsigma) d_{\beta}^{\prime} T_{1}\left(\lambda_{1}^{\beta^{\prime}}-\theta_{s_{1}}^{\prime}-\lambda_{s_{2}}^{\beta^{\prime}} \theta_{s_{2}}^{\prime}\right) \Upsilon \text {, } \\
& b_{9, l}^{\prime}=(1-\varsigma) T_{1}\left(\lambda_{1}^{l^{\prime}}-\theta_{s_{1}}^{\prime}-\lambda_{s_{2}}^{l^{\prime}} \theta_{s_{2}}^{\prime}\right) \Upsilon \text {, }
\end{aligned}
$$




$$
\begin{aligned}
& b_{10, \beta}=-(1-\varsigma) T_{1}\left(\lambda_{1}^{\beta}-\theta_{s_{1}}-\lambda_{s_{2}}^{\beta} \theta_{s_{2}}\right) \Upsilon, \\
& b_{10, l}=(1-\varsigma) T_{1}\left(\lambda_{1}^{l}-\theta_{s_{1}}-\lambda_{s_{2}}^{l} \theta_{s_{2}}\right) \Upsilon, \\
& b_{10, \beta}^{\prime}=-\varsigma\left(\delta_{\beta}^{\prime}-\xi_{\beta}^{\prime} \theta_{1}^{\prime}+d_{\beta}^{\prime 2}\left\{\delta_{\beta}^{\prime}-\left(H_{\beta}^{\prime}+\xi_{\beta}^{\prime}\right) \theta_{1}^{\prime}\right\}\right), \\
& b_{10, l}^{\prime}=\varsigma d_{l}^{\prime} S_{l}^{\prime} \theta_{1}^{\prime}, \quad b_{11, l}=-\varsigma d_{l} S_{l} \theta_{2}, \\
& b_{11, \beta}=-\varsigma\left(\zeta_{\beta}-\xi_{\beta} \theta_{2}+d_{\beta}^{2}\left\{\zeta_{\beta}-\left(H_{\beta}+\xi_{\beta}\right) \theta_{2}\right\}\right), \\
& b_{11, \beta}^{\prime}=(1-\varsigma) d_{\beta}^{\prime} T_{2}\left(\lambda_{2}^{\beta^{\prime}}-\theta_{s_{1}}^{\prime}-\lambda_{s_{2}}^{\beta^{\prime}} \theta_{s_{2}}^{\prime}\right) \Upsilon, \\
& b_{11, l}^{\prime}=(1-\varsigma) T_{2}\left(\lambda_{2}^{l^{\prime}}-\theta_{s_{1}}^{\prime}-\lambda_{s_{2}}^{l^{\prime}} \theta_{s_{2}}^{\prime}\right) \Upsilon, \\
& b_{12, \beta}=-(1-\varsigma) T_{2}\left(\lambda_{2}^{\beta}-\theta_{s_{1}}-\lambda_{s_{2}}^{\beta} \theta_{s_{2}}\right) \Upsilon, \\
& b_{12, l}=(1-\varsigma) T_{2}\left(\lambda_{2}^{l}-\theta_{s_{1}}-\lambda_{s_{2}}^{l} \theta_{s_{2}}\right) \Upsilon, \\
& b_{12, \beta}^{\prime}=-\varsigma\left(\zeta_{\beta}^{\prime}-\xi_{\beta}^{\prime} \theta_{2}^{\prime}+d_{\beta}^{\prime 2}\left\{\zeta_{\beta}^{\prime}-\left(H_{\beta}^{\prime}+\xi_{\beta}^{\prime}\right) \theta_{2}^{\prime}\right\}\right), \\
& b_{12, l}^{\prime}=\varsigma d_{l}^{\prime} S_{l}^{\prime} \theta_{2}^{\prime},
\end{aligned}
$$

Elements of matrix $\mathcal{C}$ if $P$ waves are incident.

$$
\begin{aligned}
& c_{1,1}=-b_{1, \beta}, \quad c_{2,1}=-b_{2, \beta}, \quad c_{3,1}=b_{3, \beta}, \\
& c_{4,1}=b_{4, \beta}, \quad c_{5,1}=-b_{5, \beta}, \quad c_{6,1}=-b_{6, \beta}, \\
& c_{7,1}=b_{7, \beta}, \quad c_{8,1}=b_{8, \beta}, \quad c_{9,1}=-b_{9, \beta}, \\
& c_{10,1}=b_{10, \beta}, \quad c_{11,1}=-b_{11, \beta}, \quad c_{12,1}=b_{12, \beta} .
\end{aligned}
$$

Also,

$$
\begin{aligned}
J_{\beta}= & \left(a_{11}-\frac{2}{3} G_{s_{1}}+a_{12} \lambda_{1}^{\beta}+a_{13} \lambda_{2}^{\beta}+a_{14} \lambda_{s_{2}}^{\beta}\right), \\
M_{\beta}= & a_{14}+a_{24} \lambda_{1}^{\beta}+a_{34} \lambda_{2}^{\beta}+\left(a_{44}-\frac{2}{3} G_{s_{2}}\right) \lambda_{s_{2}}^{\beta}, \\
& \delta_{\beta}=a_{12}+\lambda_{1}^{\beta} a_{22}+\lambda_{2}^{\beta} a_{23}+\lambda_{s_{2}}^{\beta} a_{24}, \\
& \zeta_{\beta}=a_{13}+\lambda_{1}^{\beta} a_{23}+\lambda_{2}^{\beta} a_{33}+\lambda_{s_{2}}^{\beta} a_{34}, \\
& J=a_{11}-\frac{2}{3} G_{s_{1}}+a_{12}+a_{13}+a_{14}, \\
& K_{1}=a_{12}+a_{22}+a_{23}+a_{24}, \\
& L_{1}=a_{13}+a_{23}+a_{33}+a_{34}, \\
& N_{1}=a_{14}+a_{24}+a_{34}+\left(a_{44}-\frac{2}{3} G_{s_{2}}\right), \\
& \xi_{\beta}=J+K_{1} \lambda_{1}^{\beta}+L_{1} \lambda_{2}^{\beta}+N_{1} \lambda_{s_{2}}^{\beta}, \\
& H_{\beta}=2 G_{s_{1}}+2 G_{s_{2}} \lambda_{s_{2}}^{\beta}, \\
& S_{l}=2 G_{s_{1}}+2 G_{s_{2}} \lambda_{s_{2}}^{l}, \\
& d_{m}=s_{m z} / s, \quad \Upsilon=\omega / s,
\end{aligned}
$$

where $s_{m z}$ corresponds to vertical slowness of all propagating waves.

\section{References}

Adamo F, Andria G, Attivissimo F and Giaquinto N 2004 An acoustic method for soil moisture measurement; IEEE Trans. Inst. Meas. 53 891-898.
Arora A and Tomar S K 2007 Elastic waves at porous/porous elastic half spaces saturated by two immiscible fluids; $J$. Porous Media 8 751-768.

Arora A, Bala N and Tomar S K 2016 A mathematical model for wave propagation in a composite solid matrix containing two immiscible fluids; Acta Mech. 227 1453-1467.

Biot M A 1956a Theory of propagation of elastic waves in a fluid saturated porous solid: I. Low frequency range; $J$. Acoust. Soc. Am. 28 168-178.

Biot M A 1956b Theory of propagation of elastic waves in a fluid saturated porous solid: II. Higher frequency range; J. Acoust. Soc. Am. 28 179-191.

Biot M A 1962 Mechanincs of deformation and acoustic propagation in porous media; J. Appl. Phys. 33 1482-1498.

Blum A, Flammer I, Friedli T and Germann P 2004 Acoustic tomography applied to water flow in unsaturated soils; Vadose Zone J. 3 288-299.

Borcherdt R D 1977 Reflection and refraction of type-II S waves in elastic and anelastic media; Bull. Seismol. Soc. Am. 67 43-67.

Borcherdt R D 1982 Reflection-refraction of general P and type-I S waves in elastic and anelastic solids; Geophys. J. Roy. Astron. Soc. 70 621-638.

Carcione J M 2014 Wave field in real media: Wave propagation in anisotropic, anelastic, porous and electromagnetic media; 3rd edn, Elsevier Science, Amsterdam.

Deckers E, Claeys C, Atak O, Groby J P, Dazel O and Desmet W 2016 A Waves based method to predict the absorption, reflection and transmission coefficient of two dimensional rigid frame porous structures with periodic inclusions; J. Comput. Phys. 312 115-138.

Denneman A I M, Drijkoningen G G, Smeulders D M J and Wapenar K 2002 Reflection and transmission of waves at a fluid/porous medium interface; Geophysics 67 282-291.

Deresiewicz H and Rice J T 1962 The effect of boundaries on wave propagation in a liquid filled porous solid-III: Reflection of plane waves at a free plane boundary; Bull. Seismol. Soc. Am. 52 595-626.

Deresiewicz H and Skalk R 1963 On uniqueness in dynamic poroelasticity; Bull. Seismol. Soc. Am. 53 793-799.

Deresiewicz H and Levy A 1967 The effect of boundaries on wave propagation in a liquid filled porous solid-X: Transmission through a stratified medium; Bull. Seismol. Soc. Am. 57 381-391.

Dutta N C, and Ode H 1983 Seismic reflections from a gas water contact; Geophysics 48 148-162.

Geertsma J and Smith D C 1961 Some aspects of elastic wave propagation in a fluid saturated porous solids; Geophysics 26 169-181.

Gorodetskaya N S 2005 Wave reflection from the free boundary of porous elastic liquid saturated half space; Int. J. Fluid Mech. Res. 32 327-339.

Hajra S and Mukhopadhyay A 1982 Reflection and refraction of seismic waves incident obliquely at the boundary of a liquid saturated porous solid; Bull. Seismol. Soc. Am. 72 $1509-1533$.

Krebes E S 1983 The viscoelastic reflection/transmission problem: two special cases; Bull. Seismol. Soc. Am. 73 1673-1683.

Kumar M and Sharma M D 2013 Reflection and transmission of attenuated waves at the boundary between two dissimilar poroelastic solids saturated with two 
immiscible viscous fluids; Geophys. Prospect. 61 10351055.

Leclaire P, Cohen-Tenoudji F and Aguirre-Puente J 1994 Extension of Biot's theory of wave propagation to frozen porous media; J. Acoust. Soc. Am. 96 3753-3768.

Lo W C, Sposito G and Majer E 2005 Wave propagation through elastic porous media containing two immiscible fluids; Water Resour. Res. 41 1-20.

Rubino J G, Ravazzoli C L and Santos J E 2006 Reflection and transmission of waves in composite porous media: A quantification of energy conversions involving slow waves; J. Acoust. Soc. Am. 120 2425-2436.

Santos J E, Corbero J M, Ravazzoli C L and Hensley J L 1992 Reflection and transmission coefficients in fluid saturated porous media; J. Acoust. Soc. Am. 91 1911-1923.

Sharma M D and Saini T 1992 Pore alignment between two dissimilar saturated poroelastic media: reflection and refraction at the interface; Int. J. Solids Struct. 29 13611377.

Sharma M D 2004 3-D wave propagation in a general anisotropic poroelastic medium: Reflection and refraction at an interface with fluid; Geophys. J. Int. 157 947-958.

Sharma M D 2005 Propagation of inhomogeneous plane waves in dissipative anistropic poroelastic solids; Geophys. J. Int. 163 981-990.

Corresponding editor: M RADHAKRISHNA
Sharma M D 2008 Wave propagation across the boundary between two dissimilar poroelastic solids; J. Sound Vib. 314 657-671.

Sharma M D 2009 Boundary conditions for porous solids saturated with viscous fluid; Appl. Math. Mech. (English Edition) 30 821-832.

Tomar S K and Arora A 2006 Reflection and transmission of elastic waves at an elastic porous solid saturated by two immiscible fluids; Int. J. Solids Struct. 43 1991-2013.

Vashisth A, Sharma M D and Gogna M L 1991 Reflection and transmission of elastic waves at a loosely bounded interface between an elastic solid and liquid saturated porous solid; Geophys. J. Int. 105 601-617.

Vlastislav C and Ivan P 2005 Plane waves in viscoelastic anistropic media I. Theory; Geophys. J. Int. 161 197212.

Yeh C L, Lo W C, Jan C D and Yang C C 2010 Reflection and refraction of obliquely incident elastic waves upon the interface between two porous elastic half spaces saturated by different fluid mixtures; J. Hydrol. 395 91-102.

Zhao X and Shen H 2015 Ocean wave transmission and reflection by viscoelasic ice covers; Ocean Model. 92 $1-10$. 